\title{
Analysis of matched primary and recurrent BRCA1/2 mutation-associated tumors identifies recurrence-specific drivers
}

\section{Jennifer Shah}

University of Pennsylvania https://orcid.org/0000-0001-9908-9874

\section{Dana Pueschl}

University of Pennsylvania

Bradley Wubbenhorst

University of Pennsylvania

John Pluta

University of Pennsylvania

Kurt D.Andrea

University of Pennsylvania

Anna Hubert

University of Pennsylvania

Jake Shilan

University of Pennsylvania

Wenting Zhou

University of Pennsylvania

Adam Kraya

University of Pennsylvania

Alba Llop-Guevara

Vall d'Hebron Institut d'Oncologia

Catherine Ruan

University of Pennsylvania

\section{Violeta Serra}

Vall d'Hebron Institut d'Oncologia https://orcid.org/0000-0001-6620-1065

Judith Balmaña

Vall d'Hebron Institut d'Oncologia https://orcid.org/0000-0002-0762-6415

\section{Michael Feldman}

University of Pennsylvania

\section{Anupma Nayak}

University of Pennsylvania

\section{Kara Maxwell}


University of Pennsylvania https://orcid.org/0000-0001-8192-4202

\section{Susan Domchek}

University of Pennsylvania https://orcid.org/0000-0002-5914-7272

Katherine Nathanson ( $\nabla$ knathans@upenn.edu )

University of Pennsylvania https://orcid.org/0000-0002-6740-0901

\section{Article}

Keywords: breast cancer, ovarian cancer, recurrence, BRCA1/2, tumors

Posted Date: August 30th, 2021

DOI: https://doi.org/10.21203/rs.3.rs-745248/v1

License: (c) (i) This work is licensed under a Creative Commons Attribution 4.0 International License. Read Full License

Version of Record: A version of this preprint was published at Nature Communications on November 7th, 2022. See the published version at https://doi.org/10.1038/s41467-022-34523-y. 
Title: Analysis of matched primary and recurrent $B R C A 1 / 2$ mutation-associated tumors identifies recurrence-specific drivers

\section{Authors, affiliations, and contact information}

1 Jennifer B. Shah1, jshah413@gmail.com

2 Dana Pueschl ${ }^{1}$, dana.pueschl@pennmedicine.upenn.edu

3 Bradley Wubbenhorst ${ }^{1}$, bwubb@pennmedicine.upenn.edu

4 John Pluta1, jpluta@pennmedicine.upenn.edu

5 Kurt D'Andrea', dandreak@upenn.edu

6 Anna P. Hubert ${ }^{1}$, anna.hubert@pennmedicine.upenn.edu

7 Jake S. Shilan', shilanjake@gmail.com

8 Wenting Zhou', wezhou@pennmedicine.upenn.edu

9 Adam A. Kraya1', krayaa@chop.edu

10 Alba Llop Guevara², allop@vhio.net

11 Catherine Ruan ${ }^{1}$, ruan.catherine@gmail.com

12 Violeta Serra ${ }^{3}$, vserra@vhio.net

13 Judith Balmaña², jbalmana@vhio.net

14 Michael Feldman ${ }^{4,6}$, michael.feldman2@pennmedicine.upenn.edu

15 Anupma Nayak ${ }^{4}$, anupma.nayak@pennmedicine.upenn.edu

16 Kara N. Maxwell ${ }^{5,6,7}$, kara.maxwell@pennmedicine.upenn.edu

17 Susan M. Domchek ${ }^{5, \overline{6}, 7}$, susan.domchek@pennmedicine.upenn.edu

18 Katherine L. Nathanson*1,6,7, knathans@upenn.edu

${ }^{1}$ Division of Translational Medicine and Human Genetics, Department of Medicine, Perelman School of Medicine at the University of Pennsylvania, Philadelphia, PA 19104, USA

2 High Risk and Familial Cancer Group, Vall d'Hebron Institut d'Oncologia, Barcelona;

Department of Medical Oncology, Hospital Vall d'Hebron, Universitat Autònoma de Barcelona, Barcelona, Spain

${ }^{3}$ Experimental Therapeutics Group, Vall d'Hebron Institut d'Oncologia, Barcelona, Spain.

${ }^{4}$ Division of Surgical Pathology, Department of Pathology and Laboratory Medicine, Perelman School of Medicine at the University of Pennsylvania, Philadelphia, PA 19104, USA

${ }^{5}$ Division of Hematology-Oncology, Department of Medicine, Perelman School of Medicine at the University of Pennsylvania, Philadelphia, PA 19104, USA

${ }^{6}$ Abramson Cancer Center, Perelman School of Medicine at the University of Pennsylvania, Philadelphia, PA 19104, USA

${ }^{7}$ Basser Center for BRCA, Perelman School of Medicine at the University of Pennsylvania, Philadelphia, PA 19104, USA

*Materials and correspondence should be addressed to K.L.N. (email: knathans@upenn.edu) 


\section{Abstract}

50 Recurrence is a major cause of death among BRCA1/2 mutation carriers with breast $(\mathrm{BrCa})$ and

51 ovarian cancers (OvCa). We performed multi-omic sequencing on 67 paired primary and

52 recurrent $\mathrm{BrCa}$ and OvCa from $27 \mathrm{BRCA} 1 / 2$ mutation carriers to identify potential recurrence-

53 specific drivers. PARP1 amplifications were identified in recurrences (FDR q=0.05), and PARP1

54 was significantly overexpressed across primary $\mathrm{BrCa}$ and recurrent $\mathrm{BrCa}$ and OvCa,

55 independent of amplification status. RNA-seq analysis found two BRCA2 isoforms, BRCA2-

56 201/Long and BRCA2-001/Short, predicted sensitive and insensitive to nonsense-mediated

57 decay, respectively. BRCA2-001/Short was expressed more frequently in recurrences and

58 associated with reduced overall survival in breast cancer (87 vs. 121 months; HR=2.535 [1.179-

59 5.45]). Loss of heterozygosity ( $\mathrm{LOH})$ status was discordant in a subset of tumors, with both $\mathrm{LOH}$

60 and lack of LOH found in recurrences. Our study revealed multiple potential drivers of recurrent

61 disease in BRCA1/2 mutation-associated cancer, improving our understanding of tumor

62 evolution and suggesting potential biomarkers.

63

64

65

66

67

68

69

70

71

72

73

74 
Pathogenic variants in BRCA1/2 are the most common cause of hereditary breast and ovarian cancer, underlying $5-10 \%$ of breast and $20 \%$ of ovarian cancers ${ }^{1,2}$. BRCA1 and BRCA2

78 function independently to maintain genomic integrity, with both playing critical roles in the

79 homologous recombination (HR) pathway of DNA repair ${ }^{3}$. The loss of BRCA1 or BRCA2 allows

80 cells to accrue mutations and genomic rearrangements facilitating a transition to cancer ${ }^{4}$.

81 Carriers of pathogenic germline variants in $B R C A 1 / 2$ have an increased risk of breast and

82 ovarian carcinomas, along with prostate and pancreatic cancers ${ }^{3,5}$.

83 Women with pathogenic variants in $B R C A 1 / 2$ are diagnosed with high-grade serous

84 ovarian carcinomas ${ }^{6}$. BRCA1 mutation carriers are predisposed to hormone receptor-negative

85 breast cancers, whereas BRCA2 mutation carriers more commonly develop hormone-positive

86 breast cancers ${ }^{3}$. Ovarian and triple-negative breast cancers tend to recur within a few years of

87 diagnosis ${ }^{6,7}$. Recurrences are usually associated with therapeutic resistance, and both recurrent

88 ovarian cancer and metastatic breast cancer are considered incurable. The standard of care in

$89 B R C A 1 / 2$ mutation-associated ovarian cancers is platinum-based chemotherapy (cisplatin,

90 carboplatin) as first line treatment, with poly (ADP-ribose) polymerase inhibitors (PARPi) used

91 increasingly in both the maintenance and metastatic settings. For BRCA1/2 mutation-

92 associated breast cancer, systemic therapy is given as appropriate to the stage and receptor

93 status of a patient's disease. PARPi are employed for late-stage breast cancer, and the

94 OlympiA trial has suggested benefit in the adjuvant setting ${ }^{8-10}$. Although tumors in $B R C A 1 / 2$

95 mutation carriers may initially respond to platinums and PARPi, many develop resistance over

96 time. Preclinical studies have demonstrated that platinum and PARPi resistance are mediated

97 by several of the same mechanisms, such as expression of MDR1 (ABCB1) efflux pumps or

98 loss of DNA damage response regulators (TP53BP1, REV7, CHD4, and PARP1) ${ }^{11-17}$.

99 However, in tumors from BRCA1/2 germline mutation carriers, the most common identified 
resistance mechanism to platinums and PARPi are reversion mutations affecting the mutant BRCA1/2 allele ${ }^{11,17-24}$.

Prior studies of matched primary and recurrent $B R C A 1 / 2$ mutation-associated breast and ovarian cancers have been limited by small numbers (3-8 patients) or focused on tumors

104 resistant to PARPi, which is not representative of current frontline therapy and resistance ${ }^{17,25-29}$.

105 At present, most BRCA1/2 mutation carriers with breast cancer do not receive PARPi prior to

106 their first recurrence. Therefore, acquired resistance mechanisms to conventional frontline

107 breast and ovarian cancer therapies are largely unknown. In addition, clinical trials have

108 evaluated the efficacy of immune checkpoint blockade on BRCA1/2 mutation-associated breast 109 and ovarian cancers, with variable objective response rates ${ }^{30-37}$. Characterizing expression of

110 checkpoint proteins in primary and recurrent BRCA1/2 mutation-associated tumors may help to 111 identify markers of response to immunomodulatory therapies.

112 To address outstanding questions about cancer recurrence in $B R C A 1 / 2$ mutation 113 carriers, we performed an analysis of paired primary and recurrent BRCA1/2 mutation114 associated tumors. The sample set consisted of 67 primary and recurrent tumors from 13 $115 B R C A 1 / 2$ germline mutation carriers with breast cancer and $14 B R C A 1 / 2$ germline mutation 116 carriers with ovarian cancer. We performed a combination of whole exome, targeted, and RNA 117 sequencing on this cohort of specimens, in addition to multiplex immunofluorescent 118 microscopy ${ }^{38,39}$. Our goal was to identify genetic, transcriptomic, and immune events that 119 underlie acquired therapeutic resistance. We hypothesized that differences between paired 120 tumors would indicate novel mechanisms of tumor evolution associated with post-treatment 121 tumor recurrence in BRCA1/2 mutation-associated cancers.

\section{$\underline{\text { Results }}$}

\section{Cohort selection and clinical characteristics}

We studied matched primary and recurrent breast cancers from 13 subjects, nine 
$B R C A 1$ and four BRCA2 pathogenic variant carriers (Table 1). All but one BRCA2 mutation carrier with breast cancer were female. Nine of 13 primary breast tumors were estrogen receptor expression negative (ER-), predominantly triple negative breast cancer (TNBC) with concordant ER- recurrences. We evaluated matched primary and recurrent ovarian cancer from

13214 women, $10 B R C A 1$ and four $B R C A 2$ pathogenic variant carriers. Most (9/14) primary ovarian

133 tumors were diagnosed at stage III or IV. As treatment for their primary tumor, all patients

134 received chemotherapy (Table 1). All 14 (100\%) ovarian cancer patients received platinums and $1354 / 14(29 \%)$ received PARPi prior to a collected recurrence. Three of 13 breast cancer patients $136(23 \%)$ received platinums and a single breast cancer patient $(1 / 13 ; 8 \%)$ received PARPi. Breast 137 cancer patients received hormonal therapy and radiation (38\% and 54\%) more frequently than 138 ovarian cancer patients (21\% and $21 \%)$. Additional clinical and sequencing details for each 139 tumor are documented in Supplementary Data 1.

Analysis of somatic mutations from whole exome and targeted sequencing

We used whole exome sequencing (WES) to assess the landscape of somatic mutations

143 in 67 paired primary/recurrent tumors and matched germline samples from 27 BRCA1/2

144 mutation carriers with breast or ovarian cancer. We also re-sequenced 44 tumors and 18

145 respective germline samples at high depth (>300x) using a custom targeted capture of 209

146 genes (Supplementary Table 1).

We assessed individual gene variants to identify shared oncogenes or tumor

148 suppressors. The majority of the cohort (53/67 tumors; $80 \%$ ) had loss of function (LoF)

149 mutations in TP53 (Fig. 1a, Supplementary Fig. 1a-c). Breast tumors without TP53 mutations

150 were mostly $B R C A 2$ mutation-associated and estrogen receptor positive $(E R+)$ in origin $(8 / 9$

151 tumors from patients 10-13). Five ovarian tumors from three patients $(17,20,25)$ also lacked

152 TP53 mutations. KMT2C was mutated in 21/67 tumors, 16 of which were ovarian tumors.

153 NOTCH1 mutations were identified exclusively in (six) breast tumors, and NF1 was mutated in 
154 six breast and ovarian tumors. One BRCA2 mutation carrier (Patient 10) had an ER+ primary

155 breast tumor and two recurrences lacking TP53 mutations, which appeared to be driven by the 156 AKT1 E17K mutation instead ${ }^{40,41}$. Gain of function (GoF) mutations were identified in PIK3CA 157 and $T B X 3$ in one breast and one ovarian tumor, respectively.

158 We next examined the global landscape of mutations by tumor. Mutational signatures, 159 were dominated by Signature 3 (BRCA1/2 inactivating mutations and defective HR) and

160 Signatures 1A/B (aging) $)^{42}$ (Fig. 1b). Sixteen of $27(60 \%)$ tumors had mutations associated with 161 Signature 4 (smoking) ${ }^{42}$. In paired tumor comparisons, tumor mutational burden (TMB) did not 162 change from primary tumor to recurrence (Supplementary Fig. 1d, Supplementary Data 2).

163 To investigate functional impact, we determined whether LoF variants accumulated in common 164 pathways across the cohort. We ranked genes by prevalence of LoF variants within primary and 165 recurrent tumor groups, then performed Gene Set Enrichment Analysis (GSEA) Preranked with 166 Hallmark Gene Sets (Fig. 1c, Supplementary Data 3) ${ }^{43}$. LoF variants from recurrences were 167 enriched for sixteen gene sets not found in primary tumor LoF variants (all FDR $q<0.25$ ). These 168 gene sets highlighted potential differences in metabolic processes (oxidative phosphorylation, 169 glycolysis), apoptosis, TP53 signaling, and pathways regulating cell growth and identity (TGF $\beta$, 170 Notch, MTORC1, KRAS).

171 To identify additional driver mutations in BRCA1/2 mutation-associated tumors, we 172 performed MutSigCV analysis on primary and recurrent tumor groups (Fig. 1d,e;

173 Supplementary Data 4) $)^{44}$. TP53 and BAGE2 were the only significantly mutated genes within 174 both tumor groups (FDR q<0.05). As BAGE2 encodes a noncoding RNA not directly targeted by 175 the exome captures used, TP53 is the only gene that can be considered significantly mutated in 176 this sample $\operatorname{set}^{45}$.

178 Analysis of BRCA1/2 loss of heterozygosity

179 Using whole exome and targeted sequencing, we assessed BRCA1/2 allele-specific loss 
of heterozygosity (LOH). First, we assessed whether somatic variants mediated LOH in our

181 sample set of tumors. We found pathogenic variants in the respective BRCA1 or BRCA2 genes

182 in 20 of 67 tumors, all with low alternative allele fraction $(<4.5 \%)$, suggesting that these

183 mutations only affected a minority of cells. Next, we used allele-specific copy number to

184 determine whether tumors underwent LOH via copy number loss of their wild-type BRCA1/2

185 allele ${ }^{46}$. Overall, 22/27 (81\%) primary tumors and 33/40 (82\%) recurrences demonstrated LOH

186 (Fig. 2a). Most primary/recurrent tumor pairs had concordant BRCA1/2 allele-specific LOH

187 status (20/27 patients; 74\%). Seven patients showed discordant LOH status when comparing

188 their primary tumor to recurrence(s). Discordant tumor LOH status was more common in

$189 B R C A 2$ mutation carriers, likely due to the increased prevalence of nonLOH ER+ breast

190 cancers $^{47}$.

191 We observed discordant LOH status more frequently in breast cancers (five $-38 \%$ ) than

192 in ovarian cancer patients (two - 14\%). Notably, three of four ER+ breast cancer patients in the

193 cohort had discordant LOH in their tumors. We noticed overlap between patients with LOH

194 transitions and those with TP53 wild-type tumors (6/7 patients $-86 \%)$. In three patients $(6,11$,

195 12), the primary tumor did not have $\mathrm{LOH}$, but first exhibited $\mathrm{LOH}$ upon recurrence (nonLOH to

196 LOH, Supplementary Fig. 2a, b). Conversely, patients 10, 13, 17, and 20 showed the opposite

197 effect: primary tumors exhibited LOH but developed into a nonLOH recurrence (LOH to

198 nonLOH, Supplementary Fig. 3a, b). This "LOH reversal" was observed in two BRCA2

199 mutation carriers with breast cancer, treated with non-platinum chemotherapy and radiation, and

200 two BRCA1 mutation carriers with ovarian cancer, both treated with platinums. Mutational

201 signatures reflected the change in HR proficiency (and potential for other drivers) among tumors

202 with LOH reversal; loss of Signature 3 was observed in patients 10, 13, and 20 (Fig. 1b). In

203 patients 10,17 , and 20 , nonLOH recurrences also had a lower HRD score compared to primary

204 tumors with LOH (Fig. 2b). For all three tumors that first developed LOH upon recurrence,

205 nonLOH primary tumors had a lower HRD score than recurrences (Fig. 2b). We did not find any 
other associations between LOH discordance and treatment history.

\section{Assessment of genomic scarring and copy number variation}

We next evaluated whether recurrent tumors showed more chromosomal instability and genomic scarring than primary tumors. Neither aneuploidy nor HRD scores (individual metrics or

212 recurrent tumors did have different scores (Supplementary Fig. 4a-e; Supplementary Data 2).

213 To identify commonly amplified and deleted genes in primary and recurrent tumors, we

214 performed GISTIC analysis, stratified by tumor type (Fig. 2c, d; Supplementary Fig. 4f, g;

215 Supplementary Fig. 5a-d; Supplementary Data 5) ${ }^{48}$. We assessed individual genes that fell 216 within significant amplifications or deletions $(90 \%$ confidence interval and FDR $q<0.05)$ and

217 were biologically relevant to breast and ovarian cancer. Both breast and ovarian tumors 218 demonstrated significant $C H D 2$ and $M Y C$ amplifications, as have been widely observed in $219 B R C A 1 / 2$ mutation-associated cancers (Supplementary Fig. 5a, b $)^{49-51}$. Breast tumors 220 demonstrated amplifications of SOX4, which has been shown to promote PI3K/AKT signaling 221 (Supplementary Fig. 5a) $)^{52}$. We observed deletions of putative breast tumor suppressor $222 R U N X 3$, DNA repair genes RAD18 and XPC, and chromatin remodelers (HDAC4/10/11 and 223 BRD1) (Supplementary Fig. 5c) ${ }^{53-55}$. Specific to ovarian tumors were amplifications in $224 A B C B 11$, an $A B C$ transporter associated with chemoresistance (Supplementary Fig. 5b $)^{56}$. 225 Other notable ovarian-specific deletions included DNA damage response genes (ERCC1/2, 226 CHEK1, FANCF), apoptotic regulators (BAX, BAD), PTEN, WEE1, and CDKN1C 227 (Supplementary Fig. 5d).

In a comparison of GISTIC segments between breast and ovarian tumors, we identified intersections ( $>50 \%$ overlap) for sixteen deletions and eight amplifications (Supplementary 231 all recurrent tumors to identify shared and distinct amplifications and deletions. Significant 
$232(90 \% \mathrm{Cl}$ and FDR $\mathrm{q}<0.05)$ deletions were more similar between primary and recurrent tumors 233 than significant amplifications (Jaccard similarity index of 0.20 vs. 0.06 ). Primary tumors had 234 amplifications in MYC and CHD2 (Fig. 2c). Recurrent tumors shared these amplifications and 235 were also uniquely enriched for amplifications of $A B C B 10$, another $A B C$ transporter associated 236 with chemotherapy resistance (Fig. 2d) ${ }^{56}$. Further, we noted recurrence-specific amplifications

237 in TGFB2, TRAF5, WNT3A, SOX4, and E2F3, suggesting that recurrences may have increased 238 dosage of growth and lineage signal pathway genes. In recurrences, unique deletions included 239 CASP9, CDKN2C, JUN, MUTYH, and RAD54L, suggesting dysregulation of cell cycle, DNA 240 damage response, and apoptosis (Supplementary Fig. 4g).

We found PARP1 to be significantly (90\% confidence interval, FDR $q=0.05)$ amplified in

242 recurrences but not primary tumors (Fig. 2d). Given the use of PARPi for treatment in BRCA1/2

243 mutation-associated tumors, we investigated further. Over half of tumors $(35 / 67,52 \%)$ in the

244 cohort had somatic gains (copy number $\geq 4$ ) or amplifications (copy number $\geq 6$ ) in PARP1

245 (Supplementary Data 6). PARP1 gains and amplifications were present in primary ovarian 246 tumors (43\%), recurrent ovarian tumors (32\%), primary breast tumors $(62 \%)$, and recurrent 247 breast tumors (78\%) (Fig. 2e). Seventy percent of all patients (19/27) in the cohort had a 248 PARP1 gain or amplification in at least one tumor (85\% of breast patients, $57 \%$ of ovarian 249 patients). Most of these patients $(11 / 19,60 \%)$ had a PARP1 gain or amplification in both 250 primary and recurrent tumors. Gains and amplifications tended to be focal and centered around 251 the PARP1 locus, rather than arm-level, underscoring a potential selection effect (Fig. $\mathbf{2 f})^{48}$. We 252 also evaluated PARP1 copy number status in TCGA PanCancer cohorts of primary breast and 253 ovarian tumors. We grouped TCGA tumors into BRCA1/2 germline mutation-associated 254 (gBRCA1/2) and HR wild-type (HR-WT) groups, as previously described (Supplementary Fig. 255 6a, Supplementary Data 7) ${ }^{47,57}$. After replicating the Sequenza analysis performed in the 256 primary/recurrent cohort, we found a high prevalence of PARP1 gains and amplifications in both 257 tumor types, with no differences based on BRCA1/2 status (Supplementary Fig. 6b, 
Supplementary Data 6).

To assess copy number variation by pathway, we next performed GSEA Preranked on lists of genes ranked by prevalence of copy number gain or loss within each tumor group (Fig.

2g; Supplementary Data 3). Primary tumors were enriched for gains in gene sets for UV response, TNF $\alpha$ signaling via NF- $\kappa \mathrm{B}, \mathrm{IFN} \alpha$ and IFN $\gamma$ response, and angiogenesis. Recurrent

263 tumors were uniquely enriched for gains in MTORC1 signaling genes and deletions in

264 Hedgehog signaling genes (consistent with our finding that recurrent tumors accumulate LoF

265 mutations in Hedgehog signaling genes).

Global transcriptomic analysis of primary and recurrent tumors

We performed RNA sequencing (RNA-seq) on 50 primary and recurrent tumors from the

269 WES cohort, adding in recurrences from four additional patients (subjects 28-31, Fig. 3a;

270 Supplementary Data 1). We also conducted RNA-seq on 12 normal breast and fallopian tube 271 samples from prophylactic surgeries in BRCA1/2 mutation carriers (subjects $32-43$, Fig. 3a,

272 Supplementary Fig. 7a, Supplementary Data 1). We used hierarchical clustering to assess

273 sample relatedness across the cohort (Supplementary Fig. 7b). In the resulting dendrogram,

274 many paired primary and recurrent tumors were not each other's closest relatives (11/19 tumor

275 pairs). In general, ER- breast tumors clustered closer to ovarian tumors than ER+ breast

276 tumors, which in turn clustered closest to normal samples. Sample relatedness appeared to be

277 most informed by ER status and tissue of origin. We further assessed sample clustering using

278 principal components (Supplementary Fig. 7c). We did not observe any clustering associated

279 with tumor origin (breast or ovarian), tumor type (primary or recurrent), or germline mutation

280 (BRCA1 vs. BRCA2).

281 To identify transcriptomic differences by tumor type, we compared gene expression in 282 primary and recurrent tumors to that of tissue-matched normal controls (Fig. 3b, c;

283 Supplementary Fig. 8a, b). Gene expression was highly similar between primary and recurrent 
284 tumors of the same origin; the average $\log _{2}$ (counts per million) for individual genes was highly 285 correlated between breast $\left(R^{2}=0.972\right)$ and ovarian $\left(R^{2}=0.965\right)$ primary and recurrent tumors. 286 ASPM, assembly factor for spindle microtubules, was highly expressed across all ovarian and 287 breast tumors (Fig. 3b, c; Supplementary Fig. 8a, b). Genes with the highest log-fold changes 288 in breast cancer included CENPF, CDK1, TOP2A, and MKI67 (Fig. 3b, Supplementary Fig. 289 8a). Among the genes with the highest log-fold changes in ovarian cancer were BIRC5, MYBL2, 290 RERGL, CRISP2, and ZCCHC12 (Fig. 3c, Supplementary Fig. 8b) ${ }^{58}$.

291 As the average $\log _{2}$ (counts per million) for individual genes were similar between breast 292 and ovarian tumors overall $\left(R^{2}=0.934\right)$, we combined all $B R C A 1 / 2$ mutation-associated tumors 293 to identify the major transcriptomic differences compared to normal tissues. Using GSEA, we 294 found enrichment of 25 Hallmark gene sets (all adj. p<0.01, Fig. 3d, Supplementary Data 8). 295 The identified gene sets included several pathways also enriched for copy number gains (Fig. 296 2g): IFN $\alpha$ and IFN $\gamma$ signaling, MTORC1 signaling, response to UV light, and TNF $a$ signaling via 297 NF- $k$ B. Increased expression of these gene sets was also identified in GSEA stratified by tumor 298 type (Supplementary Fig. 8c, d, Supplementary Data 8).

To identify transcriptomic trends driving tumorigenesis, we next interrogated genes that 300 were significantly different $\left(\mid \log _{2}(\right.$ fold change $) \mid>1$ and adj. $\left.p<0.05\right)$ in tumor vs. normal tissue. 301 Hierarchical clustering identified two modules: 1) 4715 genes with low expression in tumors; and 302 2) 2492 genes with high expression in tumors (Supplementary Fig. 8e, Supplementary Data 303 9). Low expression genes (module 1) were enriched for transcription factor binding motifs from 304 lineage-related transcription factors (Forkhead and Homeobox) and myogenic regulators $305 M E F 2 A$ and $S R F$ (all Fisher score $\geq 7$, Z-score $\geq 10$ ) (Supplementary Data 9). Using Gene 306 Ontology (GO), we evaluated the genes in module 1. Among the top hits for transcription factor 307 binding sites lost in tumors, we identified TEF-3, a transcription factor for Hippo signaling $308\left(\mathrm{p}=1.8 \times 10^{-30}\right)$; and PARP, a predicted PARP1 binding motif $\left(\mathrm{p}=6.6 \times 10^{-29}\right)^{59}$ (Fig. 3e, 309 Supplementary Data 9). We also used GO to establish trends for genes with increased 
expression in tumors (module 2) and found enrichment of terms for mitosis and cell cycle (Fig.

$3113 f$, Supplementary Data 9). In agreement with GSEA results, we also found enrichment of

312 several immune activation terms in tumors (all $p<1 \times 10^{-7}$ ) (Fig. 3f, Supplementary Data 9).

313 PARP1 expression was significantly increased in breast and ovarian recurrences

314 compared to normal tissue $\left(\log _{2} F C=1.62\right.$, adj. $p=2.52 \times 10^{-6}$ and $\log _{2} F C=9.44$, adj. $\left.p=1.58 \times 10^{-6}\right)$

315 (Fig. 3b, c). PARP1 expression was also increased in breast primary tumors ( $\log _{2} \mathrm{FC}=1.43$, adj.

$\left.316 p=3.26 \times 10^{-5}\right)($ Supplementary Fig. 8a). Primary and recurrent ovarian tumors both showed

317 increased expression of MYBL2, for which PARP1 is a putative coactivator (Fig. 3c,

318 Supplementary Fig. $\mathbf{8 b})^{60}$. Primary and recurrent breast tumors showed increased expression

319 of MKI67 (Ki67), which has been positively correlated with PARP1 expression in other breast

320 tumor cohorts (Fig. 3b, Supplementary Fig. 8a ${ }^{61}$. Overall, PARP1 expression in all tumors

321 exceeded the mean PARP1 expression in normal tissue samples, an effect that did not depend

322 on the presence of a PARP1 gain or amplification $\left(p=3.17 \times 10^{-7}\right.$ and $p=5.01 \times 10^{-5}$ for tumors with

323 and without PARP1 gain or amplification, respectively, compared to normal tissue;

324 Supplementary Fig. 9).

Analysis of gene fusions in primary and recurrent tumors

We investigated whether gene fusions could be potential drivers in BRCA1/2 mutation-

328 associated tumors. We limited our analysis to previously described gene fusions with $>5$

329 junction spanning reads (range $5-228$ reads) found in $\geq 3$ patients. Six of $54(11 \%)$ tumors had

330 fusions involving genes for immunoglobulin light and/or heavy chains (IGL@, IGH@) (Fig. 4a,

331 b; Supplementary Data 10). Fusions of MALAT1, a IncRNA associated with poor survival in

332 metastasis in several tumor types, were recently observed at high frequency $(13 / 18,72 \%)$ in

333 ovarian cancer and TNBC patients after PARPi treatment ${ }^{62,63}$. We found MALAT1 fusions in one

334 breast and two ovarian (3/54, 5.5\%) tumors. We did not identify any fusions involving efflux

335 pump $A B C B 1$, which have been previously associated with multidrug resistance ${ }^{17,25}$. 
As mRNA isoform switching events are a well-recognized phenomenon in cancer, we evaluated differential transcript usage among primary tumors, recurrent tumors, and normal

340 tissue samples ${ }^{64}$. We identified an isoform switching event between recurrences and normal

341 tissue, involving two protein-coding BRCA2 transcripts. Overall, BRCA2 gene expression was

342 significantly higher in breast and ovarian recurrent tumors compared to normal breast and

343 fallopian tube tissue (adj. $\mathrm{p}=7.13 \times 10^{-6}$, Fig. 4c). The increase in $B R C A 2$ expression was also

344 observed in breast and ovarian recurrences when compared separately to matched normal

345 tissue (both $\operatorname{logFC} \geq 1.79$, adj. $p<1 \times 10^{-3}$ ). We found that recurrent tumors preferentially

346 expressed a shorter transcript (ENST00000380152.3 or BRCA2-001, hereafter referred to as

347 "BRCA2-001/Short," $\left.\mathrm{q}=1.7 \times 10^{-4}\right)$. In contrast, normal samples favored a longer transcript

348 (ENST00000544455.1 or BRCA2-201, hereafter referred to as "BRCA2-201/Long," $\mathrm{q}=6.0 \times 10^{-4}$ )

349 (Fig. 4d). BRCA2-201/Long has a longer 3' UTR, and is predicted to be sensitive to nonsense350 mediated decay (NMD); whereas with a shorter 3' UTR, BRCA2-001/Short is predicted to be 351 NMD-insensitive (Fig. 4e $)^{65}$. The differing region of the BRCA2 3' UTR contains binding sites for 352 RNA binding proteins ELAVL1 (HuR) and PABPC1 (both FDR $q<0.01$, Supplementary Fig. 353 10a), based on RNA immunoprecipitation sequencing (RIP-seq) experiments in human B cells 354 (GEO Accession GSE35585). The presence of HuR binding sites in pre-mRNA introns and 3' 355 UTR has been directly associated with increased mRNA stability ${ }^{66}$. PABPC1 has been to 356 shown to inhibit NMD in mRNA transcripts with long $3^{\prime} \mathrm{UTRs}^{67,68}$. Of note, expression of

$357 P A B P C 1$ and ELAVL1 genes was significantly higher in tumors compared to normal tissue 358 (module 2, Supplementary Data 9).

359 We observed that expression of each BRCA2 isoform was mutually exclusive in 64 of 66 360 (97\%) RNA-seq samples, with significant association between sample group (normal, primary 361 tumor, or recurrence) and isoform usage ( $p=0.001$, Fig. 4f, Supplementary Data 11). Across all 
362 tumor samples, expression of BRCA2-001/Short was slightly more common in breast tumors $363(10 / 20 ; 50 \%)$ than in ovarian tumors $(14 / 34 ; 42 \%)$. BRCA2-001/Short was expressed in 8/11 $364(73 \%)$ breast recurrences compared to $12 / 23(52 \%)$ ovarian recurrences. In general, BRCA2365 001/Short was expressed more frequently in BRCA1 mutation-associated tumors $(21 / 42 ; 50 \%)$ 366 than in BRCA2 mutation-associated tumors (3/12; 25\%). This difference was largest within the 367 recurrence group; BRCA2-001/Short was expressed in 18/27 (67\%) BRCA1 mutation368 associated recurrences compared to 2/7 (29\%) BRCA2 mutation-associated recurrences, but 369 non-significant. There were no apparent associations with treatment history or LOH status.

370 To validate these results, we assessed BRCA2 isoform expression in an independent 371 cohort of $42 B R C A 1 / 2$ mutation-associated primary breast and ovarian tumors (Supplementary

372 Data 1, Supplementary Fig. 10b). As in our discovery set, primary tumors in the validation

373 cohort more commonly expressed BRCA2-201/Long (33/42; 79\%, Supplementary Data 11).

374 Expression of the shorter isoform BRCA2-001/Short was similarly uncommon in breast vs.

375 ovarian primary tumors $(28 \%, 29 \%)$ and in BRCA1 vs. BRCA2 mutation-associated primary 376 tumors $(28 \%, 31 \%)$.

377 Next, we tested whether expression of BRCA2-001/Short affected overall survival across 37867 BRCA1/2 mutation carriers (from discovery and validation cohorts) for whom we had survival 379 data and RNA sequenced from at least one tumor (Supplementary Data 11). Survival time for 380 breast cancer patients did not have a significant association with ER status ( $p=0.16)$, age at 381 diagnosis $(p=0.47)$, tumor stage at diagnosis $(p>0.74)$, or patient recurrence status $(p=0.58)$

382 (Supplementary Data 11). Out of 34 breast cancer patients assessed, those with BRCA2-

$383001 /$ Short expression in any tumor had significantly worse overall survival compared to patients 384 whose tumors did not express this alternative isoform $(p=0.017, \mathrm{HR}=2.535,95 \% \mathrm{Cl} 1.179-5.45)$

385 (Fig. 4g). Median overall survival was nearly three years shorter for patients whose tumor(s) 386 expressed BRCA2-001/Short ( 87 months, $95 \% \mathrm{Cl} 71.2$ - 121) compared to those whose tumors 387 did not $(121$ months, $95 \% \mathrm{Cl} 100.0$ - 159). Expression of this shorter BRCA2 isoform was not 
correlated with overall survival in 33 ovarian cancer patients after adjustment for patient recurrence status (Supplementary Fig. 10c).

\section{Analysis of DNA Damage Response and Immune Checkpoint Proteins}

We next assessed whether PARP1 copy number gains and mRNA expression translated 393 to high PARP1 protein expression. We performed immunohistochemistry (IHC) for PARP1 on 394 three tissue microarrays (TMAs) containing 23 primary and recurrent $B R C A 1 / 2$ mutation395 associated tumors from our sequencing cohort (Supplementary Fig. 11a-d, Supplementary

396 Data 1, Supplementary Methods). PARP1 protein was highly expressed across the cohort of 397 tumors (mean $\mathrm{H}$-score 252.5, median $\mathrm{H}$-score 276.4) with no significant differences by tumor 398 type (Fig. 5a). As in the RNA-seq results, high PARP1 expression did not depend on the 399 presence of a PARP1 gain or amplification (Fig. 5b). However, the only tumor with a PARP1 400 copy number loss had a relatively low $\mathrm{H}$-score of 112 . Overall, our results from RNA-seq and 401 IHC suggest that PARP1 is highly expressed in BRCA1/2 mutation-associated breast and 402 ovarian cancers.

403 Next, we assessed DNA damage markers and immune checkpoint proteins using the 404 CODEX platform (Supplementary Fig. 12a-d, Supplementary Table 2, Supplementary 405 Methods). We found no significant differences in percent of RAD51+ tumor cells between 406 primary and recurrent tumors (Supplementary Fig. 13a, b). However, we found that the 407 percent of (tumor and stromal) RAD51+ cells trended higher in tumors expressing BRCA2408 001/Short compared to those expressing BRCA2-201/Long (Fig. 5c, d). We found no 409 differences in percent of (tumor and stromal) CTLA-4+ or PD-1+ cells between primary and 410 recurrent tumors (Supplementary Fig. 13c-f).

\section{Tumor Evolution}

413 We present the multi-omic results from three patients for an in-depth analysis of the 
414 relationship between primary and recurrent tumors. Patient 20 was a BRCA1 mutation carrier

415 with an ovarian primary tumor and four recurrences (Fig. 6a). Over nine years during which the

416 patient received multiple lines of therapy, the tumor maintained BRCA1 LOH, copy number

417 gains in $A B C B 10 / 11$, high aneuploidy scores ( $\geq 13), M Y C$ amplifications $(C N \geq 10)$ and $P A R P 1$

418 gains $(\mathrm{CN}=4)$ (Supplementary Fig. 3a). However, BRCA2 isoform usage evolved, with her

419 primary tumor expressing BRCA2-201/Long and recurrences expressing BRCA2-001/Short.

420 Recurrence \#2 had different genomic features (including LoF mutations in TP53BP1, NF1,

421 TSC1, and ATM) suggesting evolution from a distinct clone (Supplementary Fig 3b). The

422 patient eventually progressed on PARPi, and a BRCA1 reversion was identified

423 (Supplementary Fig. 14a, b).

424 Patient 13 was a BRCA2 mutation carrier with TNBC. Her LOH primary yielded a

425 nonLOH recurrence, suggesting outgrowth of nonLOH sub-clones (Fig. 6 b). This recurrent

426 tumor was the only one that underwent LOH reversal without a concomitant decrease in HRD

427 score (Fig. 2b, left panel). The tumor also showed BRCA2 isoform switching and a MYC

428 amplification. The recurrence had copy number losses and mutations in HR and other DNA

429 damage response genes, including MUTYH, RAD54L, RAD52, and RAD50. RAD52 specifically

430 is required for survival of BRCA2-deficient cells ${ }^{69}$. TMB, aneuploidy, and HRD scores all

431 increased upon recurrence, indicating continued accumulation of genomic instability and

432 mutations.

433 For Patient 6, a BRCA1 mutation carrier, we evaluated one primary tumor and three

434 recurrences of TNBC (Fig. 6c). The recurrent tumor had allele-specific LOH and a BRCA2

435 isoform switch (Supplementary Fig. 2b). Two recurrences had copy number gains in

$436 A B C B 10 / 11$. Over the course of tumor progression, recurrences underwent loss of DNA

437 damage response and repair genes (by copy number loss or LoF mutation). These deleterious

438 alterations varied across recurrences but clustered into the same pathways. 
In this study, we evaluated multi-omic features of paired primary and recurrent tumors

443 from 27 BRCA1/2 mutation carriers, representing 13 breast cancers (primarily TNBC) and 14

444 ovarian cancers (mostly high-grade serous). Patients received a mix of chemotherapy and

445 radiation, in addition to platinums and PARPi, prior to collected recurrences. We assessed HRD,

446 aneuploidy, somatic mutations, copy number variation, and global transcription in paired

447 BRCA1/2 mutation-associated breast and ovarian tumors, focusing on features associated with

448 recurrence.

449 HRD scores and aneuploidy scores did not change significantly with recurrence,

450 consistent with prior findings that HRD scores do not increase upon post-platinum recurrence in

451 BRCA1/2 mutation-associated ovarian cancer $^{27}$. We also did not observe a significant increase

452 in TMB. Although a prior observation suggested TMB increases upon recurrence post-platinum

453 therapy in BRCA1/2 mutation-associated ovarian cancers, the study included only five

454 primary/recurrent ovarian tumor pairs ${ }^{17}$. Consistent with prior studies of ovarian cancers, our

455 results suggest that $B R C A 1 / 2$ mutation-associated malignancies accrue most of their genomic

456 scarring and mutations early in tumorigenesis, and increasing genomic instability is not a major

457 factor driving recurrence. TP53 was the only established cancer gene consistently mutated in

458 primary and recurrent tumors, necessary to allow cell cycle progress in genomically unstable

459 tumors ${ }^{29,47,70-73}$. We found that ER+ BRCA2 mutation-associated breast tumors, as well as

460 tumors with discordant LOH (primary vs. recurrence), were less likely to have TP53 mutations.

461 We found that PARP1 was gained or amplified across all tumor groups. However,

462 PARP1 amplifications only reached statistical significance (per GISTIC) in recurrent tumors and 463 were observed most frequently $(78 \%)$ in breast cancer recurrences. We identified up to 8-fold 464 amplifications centering on a minimal common region of chr1:226,474,131-227,148,258, which 465 fully encompasses only three other genes (ITPKB, PSEN2, STUM) in addition to PARP1. In our 
and the TCGA cohorts of primary tumors, we found a similar frequency of PARP1 gains and

467 amplifications across gBRCA1/2 and sporadic HR-WT breast and ovarian tumors. Variation in

468 methods likely accounts for the slightly different prevalence of PARP1 amplification reported in 469 cBioPortal for TCGA PanCancer ovarian (53\%) and breast $(74 \%)$ cohorts $^{74}$. We also observed 470 over two-fold increases for PARP1 expression in primary and recurrent breast tumors, as well

471 as ovarian recurrences, independent of PARP1 copy number. Our results agree with prior

472 reports of high PARP1 expression in unselected ovarian and hormone receptor-negative breast

473 cancers $^{75-77}$. Increased nuclear PARP1 protein has also been correlated with decreased

474 relapse-free and overall survival in breast cancer and $\mathrm{AML}^{78,79}$. In this study, we present the first

475 evidence of $P A R P 1$ overexpression as a near-ubiquitous feature of primary and recurrent

$476 B R C A 1 / 2$ mutation-associated tumors, corroborated at the protein level. We propose that

477 PARP1 copy number gains represent a common (but not exclusive) route to PARP1

478 overexpression, and that such gains are common in breast and ovarian tumors regardless of

$479 B R C A 1 / 2$ status. Increased levels of PARP1 protein may stoichiometrically dilute PARPi, which

480 trap PARP1/2 complexes on DNA for synthetic lethality ${ }^{80-82}$. Further, PARP1 overexpression

481 may promote PARP1-dependent alternative DNA repair pathways and transcriptional programs

482 to enhance tumor cell survival ${ }^{83,84}$. PARP1 amplifications and overexpression should be

483 explored as biomarkers of therapeutic response or clinical outcomes in recurrences in patients 484 treated with PARPi ${ }^{85-88}$.

485 We identified several significant differences between primary and recurrent tumors with 486 respect to somatic mutations, copy number variation, and gene expression. Several cancer487 associated gene sets were exclusively enriched for LoF mutations within breast and ovarian 488 recurrences, including those for apoptosis; glycolysis; P53, TGF $\beta$, Notch, and MTORC1 489 signaling. Breast and ovarian recurrences had distinct copy number profiles, despite having 490 similar HRD and aneuploidy scores. Notably, recurrences of both type had $A B C B 10$ 491 amplifications, suggesting a shared mechanism of chemoresistance only observed in vitro 
492 previously ${ }^{56}$. Hedgehog signaling genes accumulated LoF variants and copy number deletions

493 exclusively within recurrences, suggesting that Hedgehog signaling is required for primary

494 tumorigenesis (as in sporadic breast and ovarian cancers) but dispensable or dysregulated in

495 recurrence ${ }^{89,90}$. Ultimately, the genetic and transcriptomic features identified as unique to

496 recurrences could represent drivers and therapeutic targets for late-stage $B R C A 1 / 2$ mutation-

497 associated tumors.

498 We also assessed LOH status in matched tumors to determine whether BRCA1/2

499 deficiency varied over the course of tumor evolution. Seven pairs of primary and recurrent

500 tumors demonstrated changes in allele-specific LOH status, more commonly in breast cancer.

501 We observed the highest frequency of nonLOH tumors (50\% of tumors, $100 \%$ of patients) in

502 BRCA2 mutation-associated breast cancers, as has been previously reported ${ }^{47}$. We also found

503 that $\mathrm{LOH}$ discordance was more common in patients with $\mathrm{ER}+$ breast cancer and those without

504 somatic TP53 LoF mutations. Three breast cancers (one BRCA1, two BRCA2) developed allele-

505 specific LOH upon recurrence, associated with a significant increase in HRD score.

506 Interestingly, two breast (both $B R C A 2$ ) and two ovarian (both $B R C A 1$ ) tumors underwent an

507 apparent reversal of allele-specific LOH, reflected by lower HRD scores in nonLOH recurrences.

508 Prior studies have suggested LOH reversal occurs in a subset of BRCA1 mutation-associated

509 ovarian cancers after neoadjuvant platinum treatment ${ }^{29,72,91}$. We observed the same, and we

510 also identified LOH reversal in BRCA2 mutation-associated breast cancers treated with non-

511 platinum chemotherapy and radiation. NonLOH recurrences likely arose from clones resistant to

512 DNA damaging agents, although no specific pattern was observed with treatment. Our results

513 suggest that a few tumors evolve towards HR proficiency (as evidenced by nonLOH

514 recurrences) but most evolve towards HR deficiency (as evidenced by recurrences with LOH).

515 We also assessed transcriptomic differences in primary and recurrent tumors and found

516 two different isoforms of BRCA2. In silico predictions suggest that BRCA2-001/Short and

517 BRCA2-201/Long differ in regulation of mRNA stability and/or localization, as dictated by NMD 
518 sensitivity and RNA binding proteins ${ }^{64,65}$. Expression of BRCA2-001/Short was significantly

519 more common in recurrent tumors and further enriched among breast tumors and BRCA1

520 mutation carriers. We hypothesize that the isoform switch may represent a mechanism by

521 which $B R C A 1$ mutation-associated tumors can modulate $B R C A 2$ transcript stability to retain $\mathrm{HR}$

522 function and enhance tumor survival. In nonLOH BRCA2 mutation-associated tumors,

523 expression of the shorter isoform could also protect mRNA expression from the wild-type

$524 B R C A 2$ allele. Our survival analysis offers further support for a correlation between BRCA2

525 isoform expression and clinical outcomes. We found that breast cancer patients lacking BRCA2-

526 001/Short expression in any tumor (primary or recurrences) had significantly improved overall

527 survival (median 87 vs 121 months), suggesting it could be a prognostic biomarker. We did not

528 observe differences in survival based on isoform usage in ovarian cancer patients. In existing

529 literature, multiple mechanisms have been shown to restore BRCA2 function in platinum and

530 PARPi resistance, and isoform switching could represent an additional mechanism ${ }^{23,24,92-94}$. This

531 hypothesis is also supported by increased RAD51 positivity (a readout of BRCA2 activity)

532 among tumors expressing BRCA2-001/Short. Taken together, our results suggest that BRCA1

533 mutation-associated tumors, as well as nonLOH BRCA2 mutation-associated tumors, could

534 benefit from enhanced stability of $B R C A 2$ mRNA. We therefore propose that $B R C A 2$ isoform

535 switching represents a novel tumorigenic driver in BRCA1/2 mutation-associated cancers.

$536 \quad$ Although this cohort is the largest of paired primary and recurrent BRCA1/2 mutation-

537 associated tumors published to date, the number of matched primary and recurrent tumors was

538 still relatively small. As a result, we were limited in our ability to identify correlations between

539 genomic or transcriptomic features and treatment, which was quite heterogeneous. The cohort

540 includes few post-PARPi recurrences, as PARPi use was relatively rare at the time of sample

541 collection. We focused our efforts on identifying similarities and differences between paired

542 tumors across the cohort. Since we employed bulk sequencing techniques, we may not fully

543 appreciate tumor heterogeneity associated with recurrent disease. We attempted to use the 
544 same FFPE block and samples for multi-omic sequencing, but it was not always possible due to 545 sample availability. For the same reason, we could not assess $B R C A 1$ promoter methylation, 546 another possible route to biallelic BRCA1 loss in BRCA1 mutation-associated tumors. However, 547 we did confirm BRCA1 expression in all nonLOH BRCA1 mutation-associated tumors with RNA548 seq. Additionally, BRCA1 promoter methylation does not always abrogate BRCA1 expression ${ }^{95-}$ $549{ }^{97}$. Further validation of key results ( $\mathrm{LOH}$ transitions, PARP1 amplifications, BRCA2 isoform 550 switching) in an independent cohort of paired primary and recurrent BRCA1/2 mutation551 associated breast and ovarian tumors will be important. Although we were able to determine 552 that the BRCA2 isoform switching event was associated with decreased survival in two 553 independent sample sets, additional cohorts are needed to confirm, and mechanistic studies 554 remain to be done to confirm functionality.

$555 \quad$ Ultimately, these results suggest key biological features of therapy-resistant recurrences, 556 thereby highlighting novel therapeutic possibilities for BRCA1/2 mutation carriers with cancer. 557 We identified allele-specific LOH transitions in $25 \%$ of patients, underlining the plasticity of $558 B R C A 1 / 2$ status after treatment with DNA damaging agents. These results suggest that that 559 sequencing the most recent tumor or circulating DNA will be most informative in planning 560 personalized treatment. Lastly, we found that BRCA2 isoform switching may be a novel pro561 tumorigenic event that appears in both breast and ovarian recurrences and is associated with 562 reduced breast cancer patient survival. Further studies are needed to assess whether these 563 mechanisms are specific to $B R C A 1 / 2$ mutation-associated breast and ovarian cancers, or also 564 observed in the context of other tumor types or other DNA repair defects. 


\section{Methods}

Acquisition of tumor, germline, and normal tissue specimens from BRCA1/2 mutation carriers

Patients gave informed consent for research use of germline DNA, tumor specimens,

570 and clinical data, under a University of Pennsylvania IRB approved protocol. Eligible patients

571 met the following criteria: (1) diagnosis of breast or ovarian cancer; (2) positive genetic test

572 result for pathogenic germline mutation in BRCA1 or BRCA2 from a Clinical Laboratory

573 Improvement Amendments (CLIA)-approved laboratory; (3) available archived germline DNA

574 from blood or saliva; (4) available formalin-fixed paraffin-embedded (FFPE) blocks from the

575 primary tumor; and (5) available FFPE blocks from at least one recurrence matching the primary

576 tumor. Matched recurrent tumor samples were identified using manual review of patient charts

577 and pathology reports, supervised by KNM and SMD. All clinical phenotypic data were collected

578 through manual chart review. Patients 1-27 met all study criteria and comprised the

579 primary/recurrent tumor cohort. An additional four patients (Patients 28-31) lacked an available

580 primary tumor sample (meeting all but criterion 4) and were included only in groupwise RNA

581 sequencing analyses.

582 We also identified $12 B R C A 1 / 2$ mutation carriers as a control group for RNA sequencing

583 analyses. Eligible patients met the following criteria: (1) positive genetic test result for

584 pathogenic germline mutation in BRCA1 or BRCA2 from a CLIA-approved laboratory; (2) no

585 prior history of cancer or cancer treatment; and (3) available FFPE blocks from prophylactic

586 mastectomy or salpingo-oophorectomy surgeries. We extracted and sequenced RNA from

587 normal breast and fallopian tube samples from these patients. Of note, fallopian tube samples

588 were used as controls for ovarian cancer, for which the tissue of origin is the fallopian tube ${ }^{98}$.

590 Sample acquisition of primary tumor validation cohorts from TCGA and Penn

591 For validation of PARP1 copy number gains, we used WES from primary breast and ovarian

592 tumors in TCGA cohorts (Supplementary Data 7). Level 1 WES binary alignment map (bam) 
593 files from TCGA Breast and Ovarian cohorts were obtained from Genomic Data Commons

594 (https://gdc.cancer.gov/) as previously described ${ }^{47,57}$. Tumors were excluded from analysis for

595 any of the following reasons: (1) failed QC; (2) somatic BRCA1/2 loss as indicated by mutation,

596 low expression, or BRCA1 promoter methylation; or (3) germline or somatic mutations in genes

597 related to homologous recombination (see Supplementary Data 7). TCGA tumors were

598 assessed for these mutations, and grouped in gBRCA1/2 or HR-WT groups, as previously

599 described and visualized in Supplementary Fig. 6a ${ }^{47,57}$.

600 For validation of BRCA2 isoform switching, we evaluated an independent cohort of 42

601 primary breast and ovarian tumors from patients with $B R C A 1 / 2$ mutations, also consented under

602 the University of Pennsylvania approved IRB protocol. Eligible patients had a diagnosis of

603 breast or ovarian cancer with available FFPE tumor tissue and positive genetic test result for

604 pathogenic germline mutation in BRCA1 or BRCA2 from a CLIA-approved laboratory. RNA

605 extraction, sequencing, and analyses were identical to those used for primary/recurrent cohort

606 samples.

607

608 Pathological review of FFPE specimens

609 FFPE tumors were collated and sectioned by the Tumor Tissue and Biospecimen Bank

610 at the University of Pennsylvania, then stained using hematoxylin and eosin. For tumor

611 samples, staining was reviewed and marked by AN to identify sections with $\geq 70 \%$ invasive

612 tumor. Normal tissue (RNA sequencing controls) was reviewed for no evidence of tumor. DNA

613 and RNA were extracted from FFPE sections or rolls of 5-10 $\mu \mathrm{m}$ thickness. RNA extractions

614 were performed within one week of sectioning to minimize degradation.

615

616 Whole exome and targeted sequencing of tumor and germline DNA

617 DNA was extracted from FFPE using standard laboratory deparaffinization, proteinase K 618 digestion, and ethanol precipitation. Germline DNA was extracted from whole blood or saliva. 
619 Germline DNA was extracted from whole blood using sucrose-based lysis of erythrocytes,

620 followed by proteinase $\mathrm{K}$ digestion and ethanol precipitation from leukocytes. Germline DNA

621 was extracted from saliva samples using Oragene kits (DNA Genotek).

622 All DNA was sheared for library preparation using a Covaris sonicator. Tumor DNA libraries

623 were prepared using the NEBNext FFPE Repair mix and NEBNext Ultra II DNA library prep kit

624 (New England Biolabs), per manufacturer's instructions. Germline DNA libraries were prepared

625 using the NEBNext Ultra DNA library prep kit (New England Biolabs), per manufacturer's

626 instructions. DNA libraries were pooled and hybridized using SureSelect Target Enrichment

627 System for Illumina Multiplex Sequencing (Agilent) and associated protocols. For WES, tumor

628 and germline libraries were hybridized to SureSelect All Exon v5, SureSelect All Exon

629 v6+COSMIC, and SureSelect All Exon v7 captures (Agilent). For targeted sequencing, tumor

630 and germline libraries were hybridized to a custom capture, which largely utilized baits from the

631 SureSelect All Exon platform (Agilent; see Supplementary Table 1 for gene list). DNA samples,

632 libraries and hybridization pools were quantified using a Qubit (ThermoFisher) and fragment

633 size was determined using a BioAnalyzer 2100 (Agilent). WES was performed using an Illumina

634 HiSeq 4000 and targeted sequencing was performed using an Illumina NovaSeq 6000. All

635 sequencing was performed with 150 paired-end reads by the University of Pennsylvania Next

636 Generation Sequencing Core.

638 RNA sequencing of tumor and normal RNA

639 Tumor RNA was extracted from the same FFPE tumor blocks from which DNA was

640 extracted. Tumor and normal RNA samples were extracted from FFPE with the RNeasy FFPE

641 kit (Qiagen). To preserve RNA integrity, RNA samples were extracted within one week of

642 sectioning and stored at $-80^{\circ} \mathrm{C}$. We used the TruSeq RNA Exome platform (Illumina) for library

643 preparation and hybridization capture, per manufacturer protocols. Sequencing was performed 
644 with 150 paired-end reads using an Illumina HiSeq 4000 at the University of Pennsylvania Next

645 Generation Sequencing Core.

646

647 Bioinformatic analysis of DNA and RNA sequencing

648 Fastq files from whole exome and targeted sequencing were aligned to the hg19 build of

649 the human genome using the Burrows-Wheeler Aligner (BWA v.0.7.17-r1188) ${ }^{99}$. The resulting

650 bam files were processed according to Genome Analysis Toolkit (GATK v3.7) best practices

651 (picardtools v2.20.7) ${ }^{100}$. WES achieved a mean depth of 98x in tumor samples (median depth

$65287 x$ ) and 93x in germline samples (median depth 94x). Targeted sequencing achieved a mean

653 depth of 332x in tumor samples (median depth 304x) and 317x in germline samples (median

654 depth 316x).

655 Fastq files from RNA sequencing were aligned using STAR aligner (v2.7.2a) and gene

656 annotations from the GENCODE Human Release 19 reference assembly

657 (https://www.gencodegenes.org/human/release 19.html ) ${ }^{101}$. RNA sequencing achieved a mean

658 depth of 67.6 million reads (median 55.6 million reads) for tumor and normal samples.

659 Alignment and quantification of transcripts was performed using StringTie (v2.1.3b) to generate

660 abundance files in Ballgown readable format ${ }^{102}$. We used Tximport (v1.16.1) to quantify

661 transcript abundance from t_data.ctab files, generating "lengthScaledTPM" counts by gene ${ }^{103}$.

662 Next, we used EdgeR (v3.30.3) to generate, filter, and normalize counts per million (cpm) ${ }^{104}$.

663 Briefly, gene-level transcripts per million were used to create three separate DGELists: one for

664 all samples, one for ovarian tumor and normal fallopian tube samples, and one for breast tumor

665 and normal breast samples. All DGELists were filtered to include only genes for which

666 transcripts were detected in $\geq 2$ or $\geq 3$ samples, depending on the size of the smallest biological

667 group. Filtered DGELists were then normalized using the trimmed mean of M values (TMM)

668 method $^{105}$. Filtered, normalized DGELists were used to generate filtered, normalized cpm for

669 each of the three comparisons. We assessed the distribution of filtered, normalized cpm across 
670 the cohort to identify any outlying samples (Supplementary Fig. 7a). Filtered, normalized cpm

671 were used for all cohort-level and tumor-specific RNA-seq analyses, except for detection of

672 isoform switching.

673

674 Identification, filtering, and analysis of somatic variants

675 We used a union of MuTect2 (v4.1.8.1), Strelka2 (v2.9.2), VarDictJava (v1.5.1), and

676 VarScan2 (2.4.4) to call somatic variants from whole exome and targeted sequencing of

677 matched tumor and germline DNA ${ }^{106-109}$. VarDictJava and VarScan2 were used to call and filter

678 germline variants ${ }^{106,109}$. Each patient's germline variant calls were checked to ensure detection

679 of the same pathogenic $B R C A 1 / 2$ variant reported in their genetic test results. All variants were

680 annotated with ANNOVAR (October 2019 release) ${ }^{110}$. We limited our analysis of mutational

681 signatures to variants called by MuTect2 and with alternative allele depth of $\geq 10$ reads.

682 Mutational signatures were assessed separately for breast and ovarian tumor cohorts using 683 deconstructSigs $(\mathrm{v} 1.8)^{42,111}$.

684 With the exception of mutational signatures, all downstream analyses were restricted to 685 exonic variants that were called by and passed filtering for at least one caller and had

686 alternative allele read depth of $\geq$ five reads. Tumor mutational burden (TMB) was calculated for

687 each tumor using the following equation: $T M B=\frac{(n \text { somatic,exonic nonsynonymous } S N V s+\text { indels })}{\text { size of } W E S \text { capture }(M b p)}{ }^{112}$. In

688 order to exclude common SNPs from further analyses, we next removed somatic variants with 689 frequency in any population (PopFreqMax) $\geq 0.01^{110}$. Using the filtered variant set, we identified 690 significantly-mutated genes within primary and recurrent tumor groups using MutSigCV $(\mathrm{v} 1.3)^{44}$.

691 Next, we identified gain of function (GoF) and loss of function (LoF) mutations in individual

692 genes. LoF mutations were identified as those that met one of the following criteria: (1)

693 frameshift mutation, (2) nonsense mutation, or (3) nonsynonymous SNV predicted to be

694 pathogenic by REVEL score $>0.5^{113}$. GoF mutations were defined as those that met all of the 
695 following criteria: (1) not a LOF mutation; (2) documentation in Catalog of Somatic Mutations in

696 Cancer (COSMIC v84, https://cancer.sanger.ac.uk/cosmic); (3) occurring in a Tier 1 oncogene

697 as defined by the Cancer Gene Census (CGC, https://cancer.sanger.ac.uk/census); and (4)

698 matching the mutation type determined to be oncogenic for that gene by CGC.

699 Pathway analysis was performed on LoF variants using Gene Set Enrichment Analysis

700 (GSEA) Preranked (v4.0.2 for Windows) ${ }^{43}$. Briefly, genes were ranked based on frequency of

701 LoF mutations in primary and recurrent tumor groups separately. A gene could be counted as

702 mutated only once per patient within each tumor group. This approach was used to prevent

703 skewing of results by patients with multiple recurrences or by individual tumors accruing distinct

704 LOF mutations in the same gene. Ranked gene lists were used as input for GSEA Preranked

705 with Hallmark gene sets (1000 permutations). Gene set enrichment was considered significant

706 using a cutoff of FDR $<0.25$.

707

708 Genome-wide and gene-level analysis of copy number variation

709 We performed allele specific copy number analysis using Sequenza (v3.0), to identify

710 segments of copy number variation (CNVs) as well as ploidy and cellularity estimates ${ }^{46}$.

711 Segments were used to calculate homologous recombination deficiency (HRD) and aneuploidy

712 scores, using custom scripts and as previously described (https://github.com/maxwell-

$713 \underline{\text { lab/HRDex }})^{47,57}$. We used GISTIC2.0 (v2.0.23, Gene GISTIC mode, with default cutoffs) to

714 identify significant CNVs at $90 \%$ confidence in tumor cohorts ${ }^{48}$. Genes were highlighted from

715 segments with residual $\mathrm{q} \leq 0.05$.BEDtools ( $\mathrm{v} 2.28 .0$ ) was used to compute Jaccard statistics for

716 similarity between $\mathrm{CNV}$ sets, limited to segments of $\geq 50 \%$ reciprocal overlap ${ }^{114}$.

717 CNV segments were binned based on total (integer) copy number using the following

718 convention: $\mathrm{CN}=0$, Deletion; $\mathrm{CN}=1$, Loss; $\mathrm{CN}=2-3$, Neutral; $\mathrm{CN}=4-5$, Gain; $\mathrm{CN} \geq 6$, Amplification.

719 Binned segments were annotated with genes using AnnotSV (v2.3) ${ }^{115}$. Genes were considered

720 to be lost or deleted if $>50 \%$ of the locus was encompassed by a copy number loss or deletion. 
721 Conversely, genes were only considered to be gained or amplified if $100 \%$ of the locus was

722 encompassed by a gain or amplification. In the case of a gene spanning several different

723 segments of $\mathrm{CNV}$, the copy number of the gene was reported as the minimal copy number of

724 the entire locus.

725 For pathway analysis of genes subject to copy number gains, genes were ranked based

726 on frequency of their presence in a segment of gain or amplification, within primary and

727 recurrent tumor groups. Similar to our GSEA Preranked analysis of variants (see above), a gene

728 could only be counted as gained once per patient per tumor group. We then repeated this

729 process to rank genes again based on frequency of copy number loss or deletion by tumor

730 group. Ranked gene lists of "gained" genes and "lost" genes were used as input for GSEA

731 Preranked with Hallmark gene sets (1000 permutations $)^{43}$. Gene set enrichment was considered

732 significant using a cutoff of FDR $<0.25$.

733

734 Detection of BRCA1/2 Biallelic Loss

735 We first checked whether BRCA1/2 biallelic loss occurred via secondary somatic

736 mutations as detected by whole exome or targeted sequencing. Specifically, we determined

737 whether LoF mutations in $B R C A 1 / 2$ were present at an alternative allele fraction of $\geq 0.25$. This

738 cutoff reflected whether pathogenic secondary mutations were present in enough of the tumor to

739 facilitate loss of the wild-type BRCA1/2 allele. Next, we assessed loss of the wild-type BRCA1

740 or BRCA2 allele via allele-specific copy number variation from Sequenza. Briefly, we used

741 tumor and germline bam files from WES for Sequenza input. Zygosity was estimated for all

742 segments of somatic copy number variation based on the number of $A$ (major) and B (minor)

743 alleles detected. Segments with zero B alleles (only A alleles present) were deemed to have

744 undergone LOH. Conversely, if at least one B allele was detected, a segment was considered

745 nonLOH (heterozygous). Tumors were deemed to have undergone loss of heterozygosity (LOH)

746 if the segment containing their pathogenic BRCA1/2 germline mutation had LOH. When 
747 possible, we confirmed proficient BRCA1/2 expression in nonLOH tumors with RNA

748 sequencing. For patients with $\mathrm{LOH}$ transitions, we assessed the allele fraction of all $B R C A 1 / 2$

749 germline variants (snps and the pathogenic variant) across tumors to further interrogate $\mathrm{LOH}$

750 calls.

751

752 Differential gene expression and gene fusion analysis

$753 \quad$ Filtered, normalized $\log _{2} \mathrm{cpm}$ were used to assess sample relatedness. We performed

754 hierarchical clustering (maximum distance, average agglomeration) to generate dendrograms.

755 Dendrograms and principal components analysis (PCA) plots were generated using the stats

756 package in base $\mathrm{R}$ (v4.0.2). Between-group fold changes were computed as the difference in

757 average $\log _{2} \mathrm{cpm}$ for a given gene between groups. Using $\log _{2} \mathrm{cpm}$ fold changes as input, we

758 ran GSEA with Hallmark gene sets using clusterProfiler (v3.16.1) and msigdbr (v7.2.1).

759 We next used the limma R package (v3.44.3) to model mean-variance separately for all-

760 tumor, ovarian-only, and breast-only DGELists. Bayesian statistics were extracted from the

761 resulting linear models to generate adjusted p-values (Benjamini-Hochberg method) and

$762 \log _{2}$ (fold change) by gene for each comparison. Significantly altered genes were extracted using

763 decideTests in limma (global method, adj.p<0.05, $\mid \log _{2}($ fold change) $\mid>1)$.

764 Significantly altered genes for all-tumor comparisons were clustered into two gene

765 modules (see Supplementary Data 9). Hierarchical clustering of genes was based on Pearson

766 coefficients from pairwise correlations (complete method). Hierarchical clustering of samples

767 was based on Spearman coefficients from pairwise correlations (complete method). A heatmap

768 was generated from the resulting dendrograms using the gplots package (v3.1.0). Gene

769 Ontology was performed and plotted for each gene module separately, using gProfiler2 (v0.2.0).

770 Enrichment of transcription factor binding motifs was performed using oPOSSUM (v3.0) Single

771 Site Analysis (http://opossum.cisreg.ca/oPOSSUM3//116. OPOSSUM analysis was conducted 
772 against JASPAR vertebrate core profiles with cutoffs for conservation score $>0.40$, oPOSSUM

773 Fisher score $\geq 7$, and oPOSSUM Z-score $\geq 10$.

$774 \quad$ Gene fusions were identified using STAR-Fusion (v1.7.0) and plots were generated

775 using FusionInspector (v2.1.0) ${ }^{117}$. We limited our analysis of gene fusions to those with $\geq 5$

776 junction-spanning reads.

778 Assessment of differential transcript usage

Abundance was re-quantified from bam files per recommendations in the Stringtie

780 manual for novel isoform detection (http://ccb.jhu.edu/software/stringtie/index.shtml?t=manual).

781 Briefly, starting from initial bam files, we ran Stringtie in merged mode (using GENCODE

782 Human Release 19 as reference) to generate a merged gene transfer format (gtf) file for the

783 cohort. We quantified abundance from initial bams using Stringtie in Ballgown mode and the

784 merged gtf file as reference. Differential transcript usage was assessed, and switch plots

785 generated, using IsoformSwitchAnalyzer (v1.10.0). The validation cohort of primary tumors was

786 analyzed for isoform switching in an independent use of this workflow. RIP-seq plots were

787 generated using UCSC Genome Browser (hg19 assembly) with the ENCODE RNA Binding

788 Proteins track corresponding to GEO Accession GSE35585. The track pictured for

789 ELAVL1/HuR in Supplementary Fig. 10a shows a peak with coordinates chr13:32,973,328-

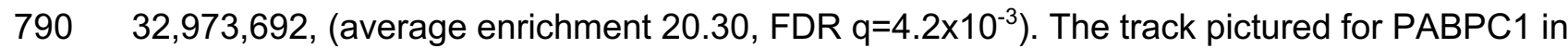

791 Supplementary Fig. 10a shows a peak with coordinates chr13:32,973,450-32,973,693

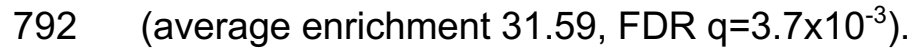

793

794 Statistical analysis

795 All paired comparisons of continuous variables (HRD scores, aneuploidy scores, TMB)

796 were tested for significance with two-sided Wilcoxon signed rank tests $(\alpha=0.05)$. For patients

797 with multiple recurrences, one recurrence was chosen at random for pairwise comparisons 
798 (such that $\mathrm{n}=27$ for each group). In the primary/recurrent cohort, groupwise differences in 799 average PARP1 copy number, PARP1 mRNA expression, and PARP1 $\mathrm{H}$-score were assessed

800 by Kruskal-Wallis test, with significant results followed by Dunn's test with Bonferroni correction $801(\alpha=0.05)$. Groupwise differences in CODEX datasets were assessed by two-sided Wilcoxon 802 rank sum test $(\alpha=0.05)$. In TCGA cohorts, groupwise differences in average PARP1 copy 803 number were assessed by two-sided t-tests $(\alpha=0.05)$. PARP1 copy number is reported for 804 primary/recurrent and TCGA tumors in Supplementary Data 6.

The adjusted $p$-value for total BRCA2 expression was generated by linear modeling with

806 limma (see above). The adjusted p-values for BRCA2 isoform usage were computed using

807 DEXSeq within isoformSwitchAnalyzer. Association between sample type and BRCA2 isoform

808 usage was determined by Mantel-Haenszel chi-squared test with continuity correction $(\alpha=0.05$, 809 1df).

810 Overall survival was defined as time from diagnosis to death or last follow-up. Survival 811 metrics were calculated based on manual chart review of the electronic health record for each 812 patient. The Penn Medicine electronic health record is indexed routinely against the National 813 Death Index, with a time delay of approximately one year. The survival analysis was limited to 81467 patients for whom we collected survival data and RNA-seq from at least one tumor (see

815 Supplementary Data 11). For the 33 ovarian cancer patients, we first used a Cox proportional

816 hazards model to control for patient age at diagnosis, tumor stage at diagnosis, and disease

817 recurrent status as confounding variables. We used the same confounding variables, in addition

818 to ER status, in multivariate Cox proportional hazards analysis of the 34 breast cancer patients.

819 In ovarian cancer patients, the only confounding variable associated with survival was disease 820 recurrent status (a logical variable indicating whether the patient's disease eventually recurred).

821 Therefore, we simplified our ovarian Cox proportional hazards model to include recurrent status

822 as the only confounding variable. In breast cancer patients, 0/4 confounding variables were 823 significantly associated with survival, so these were discarded in favor of a univariate analysis. 
824 Cox proportional hazards analyses were performed and plotted using the Survival (v3.2.7) and

825 Survminer (v0.4.8) R packages. P-values reported on the plots are those corresponding to the

826 isoform expression term itself. P-values for the models were tested for significance by Wald test

$827(\alpha=0.05,1 \mathrm{df}$ for breast, $2 \mathrm{df}$ for ovarian).

828

829 Data availability

830 Tumor WES and RNA-seq data, as well as RNA-seq from normal tissue samples, have been

831 deposited in NCBI Sequence Read Archive SUB9821849 (SRA,

832 https://www.ncbi.nlm.nih.gov/sra). Germline WES was not made publicly available to protect

833 participant privacy but can be made available upon request from the authors. TCGA data is

834 accessible via the Genomic Data Commons from the National Center Institute:

835 https://gdc.cancer.gov/. All other data are included in Supplementary Information files or will be

836 made available by the authors upon request.

837

838 Code availability

839 All code used for the study is available at https://github.com/nathanson-lab/BRCA-

840 PrimaryRecurrent. Additional code for calculating HRD and aneuploidy scores is available at

841 https://github.com/maxwell-lab/HRDex.

842

843 
844

845

846

847

848

849

850

851

852

853

854

855

856

857

858

859

860

861

862

863

864

865

866

867

868

869

870

871

872

873

874

875

876

877

878

879

880

881

882

883

884

885

886

887

888

889

890

891

892

893

894

\section{$\underline{\text { References }}$}

1. Slavin, T. P., Maxwell, K. N., Lilyquist, J., Vijai, J., Neuhausen, S. L., Hart, S. N., Ravichandran, V., Thomas, T., Maria, A., Villano, D., Schrader, K. A., Moore, R., Hu, C., Wubbenhorst, B., Wenz, B. M., D’Andrea, K., Robson, M. E., Peterlongo, P., Bonanni, B., et al. The contribution of pathogenic variants in breast cancer susceptibility genes to familial breast cancer risk. Breast Cancer 3, 22 (2017).

2. Pennington, K. P. \& Swisher, E. M. Hereditary ovarian cancer: Beyond the usual suspects. Gynecol. Oncol. 124, 347-353 (2012).

3. Roy, R., Chun, J. \& Powell, S. N. BRCA1 and BRCA2: Different Roles in a Common Pathway of Genome Protection. Nat. Rev. Cancer 12, 68-78 (2012).

4. Lord, C. J. \& Ashworth, A. BRCAness revisited. Nat. Rev. Cancer 16, 110-120 (2016).

5. Jonsson, P., Bandlamudi, C., Cheng, M. L., Srinivasan, P., Chavan, S. S., Friedman, N. D., Rosen, E. Y., Richards, A. L., Bouvier, N., Selcuklu, S. D., Bielski, C. M., Abida, W., Mandelker, D., Birsoy, O., Zhang, L., Zehir, A., Donoghue, M. T. A., Baselga, J., Offit, K., et al. Tumour lineage shapes BRCA-mediated phenotypes. Nature 571, 576-583 (2019).

6. Matulonis, U. A., Sood, A. K., Fallowfield, L., Howitt, B. E., Sehouli, J. \& Karlan, B. Y. Ovarian cancer. Nat. Rev. Dis. Prim. 2, 889-908 (2016).

7. Reddy, S. M., Barcenas, C. H., Sinha, A. K., Hsu, L., Moulder, S. L., Tripathy, D., Hortobagyi, G. N. \& Valero, V. Long-term survival outcomes of triple-receptor negative breast cancer survivors who are disease free at 5 years and relationship with low hormone receptor positivity. Br. J. Cancer 118, 17-23 (2017).

8. Tutt, A., Tovey, H., Cheang, M. C. U., Kernaghan, S., Kilburn, L., Gazinska, P., Owen, J., Abraham, J., Barrett, S., Barrett-Lee, P., Brown, R., Chan, S., Dowsett, M., Flanagan, J. M., Fox, L., Grigoriadis, A., Gutin, A., Harper-Wynne, C., Hatton, M. Q., et al. Carboplatin in BRCA1/2-mutated and triple-negative breast cancer BRCAness subgroups: The TNT Trial. Nat. Med. 24, 628-637 (2018).

9. Tung, N. M. \& Garber, J. E. BRCA1/2 testing: Therapeutic implications for breast cancer management. Br. J. Cancer 119, 141-152 (2018).

10. Tutt, A. N. J., Garber, J. E., Kaufman, B., Viale, G., Fumagalli, D., Rastogi, P., Gelber, R. D., Azambuja, E. de, Fielding, A., Balmaña, J., Domchek, S. M., Gelmon, K. A., Hollingsworth, S. J., Korde, L. A., Linderholm, B., Bandos, H., Senkus, E., Suga, J. M., Shao, Z., et al. Adjuvant Olaparib for Patients with BRCA1- or BRCA2-Mutated Breast Cancer. N. Engl. J. Med. 384, 2394-2405 (2021).

11. Lord, C. J. \& Ashworth, A. Mechanisms of resistance to therapies targeting BRCA-mutant cancers. Nature Medicine 1381-1388 (2013). doi:10.1038/nm.3369

12. Konstantinopoulos, P. A., Ceccaldi, R., Shapiro, G. I. \& D'Andrea, A. D. Homologous recombination deficiency: Exploiting the fundamental vulnerability of ovarian cancer. Cancer Discov. 5, 1137-1154 (2015).

13. Xu, G., Ross Chapman, J., Brandsma, I., Yuan, J., Mistrik, M., Bouwman, P., Bartkova, J., Gogola, E., Warmerdam, D., Barazas, M., Jaspers, J. E., Watanabe, K., Pieterse, M., Kersbergen, A., Sol, W., Celie, P. H. N., Schouten, P. C., Van Den Broek, B., Salman, A., et al. REV7 counteracts DNA double-strand break resection and affects PARP inhibition. Nature 521, 541-544 (2015).

14. Guillemette, S., Serra, R. W., Peng, M., Hayes, J. A., Konstantinopoulos, P. A., Green, M. R., Green, M. R. \& Cantor, S. B. Resistance to therapy in BRCA2 mutant cells due to loss of the nucleosome remodeling factor CHD4. Genes Dev. 29, 489-494 (2015).

15. Pettit, S. J., Rehman, F. L., Bajrami, I., Brough, R., Wallberg, F., Kozarewa, I., Fenwick, K., Assiotis, I., Chen, L., Campbell, J., Lord, C. J. \& Ashworth, A. A Genetic Screen Using the PiggyBac Transposon in Haploid Cells Identifies Parp1 as a Mediator of Olaparib Toxicity. PLoS One 8, e61520 (2013). 
16. Bunting, S. F., Callén, E., Wong, N., Chen, H.-T., Polato, F., Gunn, A., Bothmer, A., Feldhahn, N., Fernandez-Capetillo, O., Cao, L., Xu, X., Deng, C.-X., Finkel, T., Nussenzweig, M., Stark, J. M. \& Nussenzweig, A. 53BP1 Inhibits Homologous Recombination in Brca1-Deficient Cells by Blocking Resection of DNA Breaks. Cell 141, 243-254 (2010).

17. Patch, A. M., Christie, E. L., Etemadmoghadam, D., Garsed, D. W., George, J., Fereday, S., Nones, K., Cowin, P., Alsop, K., Bailey, P. J., Kassahn, K. S., Newell, F., Quinn, M. C. J., Kazakoff, S., Quek, K., Wilhelm-Benartzi, C., Curry, E., Leong, H. S., Hamilton, A., et al. Whole-genome characterization of chemoresistant ovarian cancer. Nature 521, 489494 (2015).

18. Goodall, J., Mateo, J., Yuan, W., Mossop, H., Porta, N., Miranda, S., Perez-Lopez, R., Dolling, D., Robinson, D. R., Sandhu, S., Fowler, G., Ebbs, B., Flohr, P., Seed, G., Rodrigues, D. N., Boysen, G., Bertan, C., Atkin, M., Clarke, M., et al. Circulating cell-free DNA to guide prostate cancer treatment with PARP inhibition. Cancer Discov. 7, 10061017 (2017).

19. Quigley, D., Alumkal, J. J., Wyatt, A. W., Kothari, V., Foye, A., Lloyd, P., Aggarwal, R., Kim, W., Lu, E., Schwartzman, J., Beja, K., Annala, M., Das, R., Diolaiti, M., Pritchard, C., Thomas, G., Tomlins, S., Knudsen, K., Lord, C. J., et al. Analysis of circulating cell-free DnA identifies multiclonal heterogeneity of BRCA2 reversion mutations associated with resistance to PARP inhibitors. Cancer Discov. 7, 999-1005 (2017).

20. Cheng, H. H., Salipante, S. J., Nelson, P. S., Montgomery, B. \& Pritchard, C. C. Polyclonal BRCA2 Reversion Mutations Detected in Circulating Tumor DNA After Platinum Chemotherapy in a Patient With Metastatic Prostate Cancer. JCO Precis. Oncol. (2018). doi:10.1200/PO

21. Swisher, E. M., Sakai, W., Karlan, B. Y., Wurz, K., Urban, N. \& Taniguchi, T. Secondary BRCA1 mutations in BRCA1-mutated ovarian carcinomas with platinum resistance. Cancer Res. 68, 2581-2586 (2008).

22. Norquist, B., Wurz, K. A., Pennil, C. C., Garcia, R., Gross, J., Sakai, W., Karlan, B. Y., Taniguchi, T. \& Swisher, E. M. Secondary somatic mutations restoring BRCA1/2 predict chemotherapy resistance in hereditary ovarian carcinomas. J. Clin. Oncol. 29, 3008-3015 (2011).

23. Banda, K., Swisher, E. M., Wu, D., Pritchard, C. C. \& Gadi, V. K. Somatic Reversion of Germline BRCA2 Mutation Confers Resistance to Poly(ADP-ribose) Polymerase Inhibitor Therapy. JCO Precis. Oncol. 1-6 (2018). doi:10.1200/PO.17.00044

24. Barber, L. J., Sandhu, S., Chen, L., Campbell, J., Kozarewa, I., Fenwick, K., Assiotis, I., Rodrigues, D. N., Filho, J. S. R., Moreno, V., Mateo, J., Molife, L. R., De Bono, J., Kaye, S., Lord, C. J. \& Ashworth, A. Secondary mutations in BRCA2 associated with clinical resistance to a PARP inhibitor. J. Pathol. 229, 422-429 (2013).

25. Brady, S. W., McQuerry, J. A., Qiao, Y., Piccolo, S. R., Shrestha, G., Jenkins, D. F., Layer, R. M., Pedersen, B. S., Miller, R. H., Esch, A., Selitsky, S. R., Parker, J. S., Anderson, L. A., Dalley, B. K., Factor, R. E., Reddy, C. B., Boltax, J. P., Li, D. Y., Moos, P. J., et al. Combating subclonal evolution of resistant cancer phenotypes. Nat. Commun. 8, 1231 (2017).

26. Meric-Bernstam, F., Frampton, G. M., Ferrer-Lozano, J., Yelensky, R., Perez-Fidalgo, J. A., Wang, Y., Palmer, G. A., Ross, J. S., Miller, V. A., Su, X., Eroles, P., Barrera, J. A., Burgues, O., Lluch, A. M., Zheng, X., Sahin, A., Stephens, P. J., Mills, G. B., Cronin, M. T., et al. Concordance of Genomic Alterations between Primary and Recurrent Breast Cancer. Mol. Cancer Ther. 13, 1382-1389 (2014).

27. Patel, J. N., Braicu, I., Timms, K. M., Solimeno, C., Tshiaba, P., Reid, J., Lanchbury, J. S., Darb-Esfahani, S., Ganapathi, M. K., Sehouli, J. \& Ganapathi, R. N. Characterisation of homologous recombination deficiency in paired primary and recurrent high grade 
946

947

948

949

950

951

952

953

954

955

956

957

958

959

960

961

962

963

964

965

966

967

968

969

970

971

972

973

974

975

976

977

978

979

980

981

982

983

984

985

986

987

988

989

990

991

992

993

994

995

996

serous ovarian cancer. Br. J. Cancer 1-7 (2018). doi:10.1038/s41416-018-0268-6

28. Paul, M. R., Pan, T., Pant, D. K., Shih, N. N. C., Chen, Y., Harvey, K. L., Solomon, A., Lieberman, D., Morrissette, J. J. D., Soucier-Ernst, D., Goodman, N. G., Stavropoulos, S. W., Maxwell, K. N., Clark, C., Belka, G. K., Feldman, M., DeMichele, A. \& Chodosh, L. A. Genomic landscape of metastatic breast cancer identifies preferentially dysregulated pathways and targets. J. Clin. Invest. 130, 4252-4265 (2020).

29. Sokolenko, A. P., Bizin, I. V., Preobrazhenskaya, E. V., Gorodnova, T. V., Ivantsov, A. O., lyevleva, A. G., Savonevich, E. L., Kotiv, K. B., Kuligina, E. S. \& Imyanitov, E. N. Molecular profiles of BRCA1-associated ovarian cancer treated by platinum-based therapy: Analysis of primary, residual and relapsed tumors. Int. J. Cancer 146, 1879$1888(2020)$.

30. LoRusso, P., Pilat, M. J. P., Santa-Maria, C. A., Connolly, R. M., Roesch, E. E., Afghahi, A., Han, H. S., Nanda, R., Wulf, G. M., Assad, H., Park, H., Dees, E. C., Force, J. M., Noonan, A. M., Brufsky, A., Abramson, V. G., Haley, B. B., Buys, S. S., Sharon, E., et al. Trial in progress: A phase II open-label, randomized study of PARP inhibition (olaparib) either alone or in combination with anti-PD-L1 therapy (atezolizumab) in homologous DNA repair (HDR) deficient, locally advanced or metastatic non-HER2-positive breast cancer. J. Clin. Oncol. 38, TPS1102-TPS1102 (2020).

31. Zhang, J., Shih, D. J. H. \& Lin, S. Y. Role of DNA repair defects in predicting immunotherapy response. Biomark. Res. 8, 1-8 (2020).

32. Zhang, G., Liu, C., Bai, H., Cao, G., Cui, R. \& Zhang, Z. Combinatorial therapy of immune checkpoint and cancer pathways provides a novel perspective on ovarian cancer treatment (Review). Oncol. Lett. 17, 2583-2591 (2019).

33. Matulonis, U. A., Shapira-Frommer, R., Santin, A. D., Lisyanskaya, A. S., Pignata, S., Vergote, I., Raspagliesi, F., Sonke, G. S., Birrer, M., Provencher, D. M., Sehouli, J., Colombo, N., González-Martín, A., Oaknin, A., Ottevanger, P. B., Rudaitis, V., Katchar, $\mathrm{K} ., \mathrm{Wu}, \mathrm{H}$., Keefe, S., et al. Antitumor activity and safety of pembrolizumab in patients with advanced recurrent ovarian cancer: results from the phase II KEYNOTE-100 study. Ann. Oncol. 30, 1080-1087 (2019).

34. Disis, M. L., Taylor, M. H., Kelly, K., Thaddeus Beck, J., Gordon, M., Moore, K. M., Patel, M. R., Chaves, J., Park, H., Mita, A. C., Hamilton, E. P., Annunziata, C. M., Juergen Grote, H., von Heydebreck, A., Grewal, J., Chand, V. \& Gulley, J. L. Efficacy and Safety of Avelumab for Patients With Recurrent or Refractory Ovarian Cancer Phase $1 \mathrm{~b}$ : Results From the JAVELIN Solid Tumor Trial. JAMA Oncol. 5, 393-401 (2019).

35. Domchek, S. M., Postel-Vinay, S., Im, S.-A., Park, Y. H., Delord, J.-P., Italiano, A., Alexandre, J., You, B., Bastian, S., Krebs, M. G., Wang, D., Waqar, S. N., Lanasa, M., Rhee, J., Gao, H., Rocher-Ros, V., Jones, E. V, Gulati, S., Coenen-Stass, A., et al. Olaparib and durvalumab in patients with germline BRCA-mutated metastatic breast cancer (MEDIOLA): an open-label, multicentre, phase 1/2, basket study. Lancet Oncol. 21, 1155-1164 (2020).

36. Hamanishi, J., Mandai, M., Ikeda, T., Minami, M., Kawaguchi, A., Murayama, T., Kanai, M., Mori, Y., Matsumoto, S., Chikuma, S., Matsumura, N., Abiko, K., Baba, T., Yamaguchi, K., Ueda, A., Hosoe, Y., Morita, S., Yokode, M., Shimizu, A., et al. Safety and antitumor activity of Anti-PD-1 antibody, nivolumab, in patients with platinumresistant ovarian cancer. J. Clin. Oncol. 33, 4015-4022 (2015).

37. Liu, Y. L., Selenica, P., Zhou, Q., lasonos, A., Callahan, M., Feit, N. Z., Boland, J., Vazquez-Garcia, I., Mandelker, D., Zehir, A., Burger, R. A., Powell, D. J., Friedman, C., Cadoo, K., Grisham, R., Konner, J. A., O'Cearbhaill, R. E., Aghajanian, C., Reis-Filho, J. S., et al. BRCA Mutations, Homologous DNA Repair Deficiency, Tumor Mutational Burden, and Response to Immune Checkpoint Inhibition in Recurrent Ovarian Cancer . JCO Precis. Oncol. 4, 665-679 (2020). 
997

998

999

1000

1001

1002

1003

1004

1005

1006

1007

1008

1009

1010

1011

1012

1013

1014

1015

1016

1017

1018

1019

1020

1021

1022

1023

1024

1025

1026

1027

1028

1029

1030

1031

1032

1033

1034

1035

1036

1037

1038

1039

1040

1041

1042

1043

1044

1045

1046

1047
38. Goltsev, Y., Samusik, N., Kennedy-Darling, J., Bhate, S., Hale, M., Vazquez, G., Black, S. \& Nolan, G. P. Deep Profiling of Mouse Splenic Architecture with CODEX Multiplexed Imaging. Cell 174, 968-981.e15 (2018).

39. Du, Z., Lin, J. R., Rashid, R., Maliga, Z., Wang, S., Aster, J. C., Izar, B., Sorger, P. K. \& Santagata, S. Qualifying antibodies for image-based immune profiling and multiplexed tissue imaging. Nat. Protoc. 14, 2900-2930 (2019).

40. Carpten, J. D., Faber, A. L., Horn, C., Donoho, G. P., Briggs, S. L., Robbins, C. M., Hostetter, G., Boguslawski, S., Moses, T. Y., Savage, S., Uhlik, M., Lin, A., Du, J., Qian, Y.-W., Zeckner, D. J., Tucker-Kellogg, G., Touchman, J., Patel, K., Mousses, S., et al. A transforming mutation in the pleckstrin homology domain of AKT1 in cancer. Nature 448, 439-445 (2007).

41. Chen, Y., Huang, L., Dong, Y., Tao, C., Zhang, R., Shao, H. \& Shen, H. Effect of AKT1 (p. E17K) Hotspot Mutation on Malignant Tumorigenesis and Prognosis. Frontiers in Cell and Developmental Biology 8, 1-12 (2020).

42. Alexandrov, L. B., Nik-Zainal, S., Wedge, D. C., Aparicio, S. A. J. R., Behjati, S., Biankin, A. V., Bignell, G. R., Bolli, N., Borg, A., Børresen-Dale, A. L., Boyault, S., Burkhardt, B., Butler, A. P., Caldas, C., Davies, H. R., Desmedt, C., Eils, R., Eyfjörd, J. E., Foekens, J. A., et al. Signatures of mutational processes in human cancer. Nature 500, 415-421 (2013).

43. Subramanian, A., Tamayo, P., Mootha, V. K., Mukherjee, S., Ebert, B. L., Gillette, M. A., Paulovich, A., Pomeroy, S. L., Golub, T. R., Lander, E. S. \& Mesirov, J. P. Gene set enrichment analysis: a knowledge-based approach for interpreting genome-wide expression profiles. Proc. Natl. Acad. Sci. U. S. A. 102, 15545-50 (2005).

44. Lawrence, M. S., Stojanov, P., Polak, P., Kryukov, G. V., Cibulskis, K., Sivachenko, A., Carter, S. L., Stewart, C., Mermel, C. H., Roberts, S. A., Kiezun, A., Hammerman, P. S., McKenna, A., Drier, Y., Zou, L., Ramos, A. H., Pugh, T. J., Stransky, N., Helman, E., et al. Mutational heterogeneity in cancer and the search for new cancer-associated genes. Nature 499, 214-218 (2013).

45. Ruault, M., van der Bruggen, P., Brun, M. E., Boyle, S., Roizès, G. \& De Sario, A. New BAGE (B melanoma antigen) genes mapping to the juxtacentromeric regions of human chromosomes 13 and 21 have a cancer/testis expression profile. Eur. J. Hum. Genet. 10, 833-840 (2002).

46. Favero, F., Joshi, T., Marquard, A. M., Birkbak, N. J., Krzystanek, M., Li, Q., Szallasi, Z. \& Eklund, A. K. Sequenza: allele-specific copy number and mutation profiles from tumor sequencing data. Ann. Oncol. 26, 64-70 (2014).

47. Maxwell, K. N., Wubbenhorst, B., Wenz, B. M., De Sloover, D., Pluta, J., Emery, L., Barrett, A., Kraya, A. A., Anastopoulos, I. N., Yu, S., Jiang, Y., Chen, H., Zhang, N. R., Hackman, N., D'andrea, K., Daber, R., Morrissette, J. J. D., Mitra, N., Feldman, M., et al. BRCA locus-specific loss of heterozygosity in germline BRCA1 and BRCA2 carriers. Nat. Commun. 8, 1-11 (2017).

48. Mermel, C. H., Schumacher, S. E., Hill, B., Meyerson, M. L., Beroukhim, R. \& Getz, G. GISTIC2.0 facilitates sensitive and confident localization of the targets of focal somatic copy-number alteration in human cancers. Genome Biol. 12, R41 (2011).

49. Grushko, T. A., Dignam, J. J., Das, S., Blackwood, A. M., Perou, C. M., Ridderstråle, K. K., Anderson, K. N., Wei, M. J., Adams, A. J., Hagos, F. G., Sveen, L., Lynch, H. T., Weber, B. L. \& Olopade, O. I. MYC Is Amplified in BRCA1-Associated Breast Cancers. Clin. Cancer Res. 10, 499-507 (2004).

50. Castro, E., Jugurnauth-Little, S., Karlsson, Q., Al-Shahrour, F., Piñeiro-Yañez, E., Van de Poll, F., Leongamornlert, D., Dadaev, T., Govindasami, K., Guy, M., Eeles, R. \& KoteJarai, Z. High burden of copy number alterations and c-MYC amplification in prostate cancer from BRCA2 germline mutation carriers. Ann. Oncol. 26, 2293-2300 (2015). 
1048

1049

1050

1051

1052

1053

1054

1055

1056

1057

1058

1059

1060

1061

1062

1063

1064

1065

1066

1067

1068

1069

1070

1071

1072

1073

1074

1075

1076

1077

1078

1079

1080

1081

1082

1083

1084

1085

1086

1087

1088

1089

1090

1091

1092

1093

1094

1095

1096

1097

1098
51. Taylor, R. A., Fraser, M., Livingstone, J., Melijah, S., Espiritu, G., Thorne, H., Huang, V., Lo, W., Shiah, Y.-J., Yamaguchi, T. N., Sliwinski, A., Horsburgh, S., Meng, A., Heisler, L. E., Yu, N., Yousif, F., Papargiris, M., Lawrence, M. G., Timms, L., et al. Germline BRCA2 mutations drive prostate cancers with distinct evolutionary trajectories. Nat. Commun. 8, 13671 (2017).

52. Mehta, G. A., Parker, J. S., Silva, G. O., Hoadley, K. A., Perou, C. M. \& Gatza, M. L. Amplification of SOX4 promotes PI3K/Akt signaling in human breast cancer. Breast Cancer Res. Treat. 162, 439-450 (2017).

53. Marmorstein, R. \& Zhou, M. Writers and Readers of Histone Acetylation : Structure, Mechanism, and Inhibition. Cold Spring Harb Perspect Biol 6, 1-26 (2014).

54. Li, Y. \& Seto, E. HDACs and HDAC inhibitors in cancer development and therapy. Cold Spring Harb. Perspect. Med. 6, 1-34 (2016).

55. Chen, L. F. Tumor suppressor function of RUNX3 in breast cancer. J. Cell. Biochem. 113, 1470-1477 (2012).

56. Yasui, K., Mihara, S., Zhao, C., Okamoto, H., Saito-Ohara, F., Tomida, A., Funato, T., Yokomizo, A., Naito, S., Imoto, I., Tsuruo, T. \& Inazawa, J. Alteration in Copy Numbers of Genes as a Mechanism for Acquired Drug Resistance. Cancer Res. 64, 1403-1410 (2004).

57. Kraya, A. A., Maxwell, K. N., Wubbenhorst, B., Wenz, B. M., Pluta, J., Rech, A. J., Dorfman, L. M., Lunceford, N., Barrett, A., Mitra, N., Morrissette, J. J. D., Feldman, M., Nayak, A., Domchek, S. M., Vonderheide, R. H. \& Nathanson, K. L. Genomic Signatures Predict the Immunogenicity of BRCA-Deficient Breast Cancer. (2019). doi:10.1158/10780432.CCR-18-0468

58. Wheatley, S. P. \& Altieri, D. C. Survivin at a glance. J. Cell Sci. 132, 7 (2019).

59. Ma, Y., Yang, Y., Wang, F., Wei, Q. \& Qin, H. Hippo-YAP signaling pathway: A new paradigm for cancer therapy. Int. J. Cancer 137, 2275-2286 (2015).

60. Santilli, G., Cervellera, M. N., Johnson, T. K., Lewis, R. E., lacobelli, S. \& Sala, A. PARP co-activates B-MYB through enhanced phosphorylation at cyclin/cdk2 sites. Oncogene 20, 8167-8174 (2001).

61. Bieche, I., Pennaneach, V., Driouch, K., Vacher, S., Zaremba, T., Susini, A., Lidereau, R. \& Hall, J. Variations in the mRNA expression of poly(ADP-ribose) polymerases, poly(ADP-ribose) glycohydrolase and ADP-ribosylhydrolase 3 in breast tumors and impact on clinical outcome. Int. J. Cancer 133, 2791-2800 (2013).

62. Eismann, J., Heng, Y. J., Waldschmidt, J. M., Vlachos, I. S., Gray, K. P., Matulonis, U. A., Konstantinopoulos, P. A., Charles, ', Murphy, J., Nabavi, S., Gerburg, · \& Wulf, M. Transcriptome analysis reveals overlap in fusion genes in a phase I clinical cohort of TNBC and HGSOC patients treated with buparlisib and olaparib. J. Cancer Res. Clin. Oncol. 146, 503-514 (2020).

63. Li, Z.-X., Zhu, Q.-N., Zhang, H.-B., Hu, Y., Wang, G. \& Zhu, Y.-S. MALAT1: a potential biomarker in cancer. Cancer Manag. Res. 6757-6768 (2018). doi:10.2147/CMAR.S169406

64. Vitting-Seerup, K. \& Sandelin, A. The landscape of isoform switches in human cancers. Mol. Cancer Res. 15, 1206-1220 (2017).

65. Vitting-Seerup, K., Porse, B. T., Sandelin, A. \& Waage, J. SpliceR: An R package for classification of alternative splicing and prediction of coding potential from RNA-seq data. BMC Bioinformatics 15, 81 (2014).

66. Mukherjee, N., Corcoran, D. L., Nusbaum, J. D., Reid, D. W., Georgiev, S., Hafner, M., Ascano, M., Tuschl, T., Ohler, U. \& Keene, J. D. Integrative Regulatory Mapping Indicates that the RNA-Binding Protein HuR Couples Pre-mRNA Processing and mRNA Stability. Mol. Cell 43, 327-339 (2011).

67. Fatscher, T., Boehm, V., Weiche, B. \& Gehring, N. H. The interaction of cytoplasmic 
poly(A)-binding protein with eukaryotic initiation factor $4 \mathrm{G}$ suppresses nonsensemediated mRNA decay. RNA 20, 1579-1592 (2014).

68. Behm-Ansmant, I., Gatfield, D., Rehwinkel, J., Hilgers, V. \& Izaurralde, E. A conserved role for cytoplasmic poly(A)-binding protein 1 (PABPC1) in nonsense-mediated mRNA decay. EMBO J. 26, 1591-1601 (2007).

69. Feng, Z., Scott, S. P., Bussen, W., Sharma, G. G., Guo, G., Pandita, T. K. \& Powell, S. N. Rad52 inactivation is synthetically lethal with BRCA2 deficiency. PNAS 108, 686-691 (2010).

70. Inagaki-Kawata, Y., Yoshida, K., Kawaguchi-Sakita, N., Kawashima, M., Nishimura, T., Senda, N., Shiozawa, Y., Takeuchi, Y., Inoue, Y., Sato-Otsubo, A., Fujii, Y., Nannya, Y., Suzuki, E., Takada, M., Tanaka, H., Shiraishi, Y., Chiba, K., Kataoka, Y., Torii, M., et al. Genetic and clinical landscape of breast cancers with germline BRCA1/2 variants. Commun. Biol. 3, 1-9 (2020).

71. Boyarskikh, U. A., Gulyaeva, L. F., Avdalyan, A. M., Kechin, A. A., Khrapov, E. A., Lazareva, D. G., Kushlinskii, N. E., Melkonyan, A., Arakelyan, A. \& Filipenko, M. L. Spectrum of TP53 Mutations in BRCA1/2 Associated High-Grade Serous Ovarian Cancer. Front. Oncol. 10, 1103 (2020).

72. Sokolenko, A. P., Savonevich, E. L., Ivantsov, A. O., Raskin, G. A., Kuligina, E. S., Gorodnova, T. V., Preobrazhenskaya, E. V., Kleshchov, M. A., Tiurin, V. I., Mukhina, M. S., Kotiv, K. B., Shulga, A. V., Kuznetsov, S. G., Berlev, I. V. \& Imyanitov, E. N. Rapid selection of BRCA1-proficient tumor cells during neoadjuvant therapy for ovarian cancer in BRCA1 mutation carriers. Cancer Lett. 397, 127-132 (2017).

73. Holstege, H., Joosse, S. A., Th van Oostrom, C. M., Nederlof, P. M., de Vries, A. \& Jonkers, J. High Incidence of Protein-Truncating TP53 Mutations in BRCA1-Related Breast Cancer. Cancer Res 69, 3625-3658 (2009).

74. Hoadley, K. A., Yau, C., Hinoue, T., Wolf, D. M., Lazar, A. J., Drill, E., Shen, R., Taylor, A. M., Cherniack, A. D., Thorsson, V., Akbani, R., Bowlby, R., Wong, C. K., Wiznerowicz, M., Sanchez-Vega, F., Robertson, A. G., Schneider, B. G., Lawrence, M. S., Noushmehr, H., et al. Cell-of-Origin Patterns Dominate the Molecular Classification of 10,000 Tumors from 33 Types of Cancer. Cell 173, 291-304.e6 (2018).

75. Ossovskaya, V., Koo, I. C., Kaldjian, E. P., Alvares, C. \& Sherman, B. M. Upregulation of poly (ADP-Ribose) polymerase-1 (PARP1) in triple-negative breast cancer and other primary human tumor types. Genes and Cancer 1, 812-821 (2010).

76. $\mathrm{Bi}, \mathrm{F} . \mathrm{F} ., \mathrm{Li}, \mathrm{D} . \&$ Yang, Q. Hypomethylation of ETS transcription factor binding sites and upregulation of PARP1 expression in endometrial cancer. Biomed Res. Int. 2013, 1-5 (2013).

77. Lheureux, S., Bruce, J. P., Burnier, J. V., Karakasis, K., Shaw, P. A., Clarke, B. A., Yang, S. Y. C., Quevedo, R., Li, T., Dowar, M., Bowering, V., Pugh, T. J. \& Oza, A. M. Somatic BRCA1/2 recovery as a resistance mechanism after exceptional response to poly (ADPribose) polymerase inhibition. J. Clin. Oncol. 35, 1240-1249 (2017).

78. Rojo, F., García-Parra, J., Zazo, S., Tusquets, I., Ferrer-Lozano, J., Menendez, S., Eroles, P., Chamizo, C., Servitja, S., Ramírez-Merino, N., Lobo, F., Bellosillo, B., Corominas, J. M., Yelamos, J., Serrano, S., Lluch, A., Rovira, A. \& Albanell, \& J. Nuclear PARP-1 protein overexpression is associated with poor overall survival in early breast cancer. Ann. Oncol. 23, 1156-1164 (2012).

79. Pashaiefar, H., Yaghmaie, M., Tavakkoly-Bazzaz, J., Ghaffari, S. H., Alimoghaddam, K., Momeny, M., Izadi, P., Izadifard, M., Kasaeian, A. \& Ghavamzadeh, A. PARP-1 Overexpression as an Independent Prognostic Factor in Adult Non-M3 Acute Myeloid Leukemia. Genet. Test. Mol. Biomarkers 22, 343-349 (2018).

80. Murai, J., Huang, S. N., Das, B. B., Renaud, A., Zhang, Y., Doroshow, J. H., Ji, J., Takeda, S. \& Pommier, Y. Trapping of PARP1 and PARP2 by Clinical PARP Inhibitors. 
Cancer Res. 72, 5588-5599 (2012).

81. Pommier, Y., O'Connor, M. J. \& De Bono, J. Laying a trap to kill cancer cells: PARP inhibitors and their mechanisms of action. Sci. Transl. Med. 8, 1-8 (2016).

82. Lord, C. J. \& Ashworth, A. PARP inhibitors: Synthetic lethality in the clinic. Science (80). 355, 1152-1158 (2017).

83. Ray Chaudhuri, A. \& Nussenzweig, A. The multifaceted roles of PARP1 in DNA repair and chromatin remodelling. Nat. Rev. Mol. Cell Biol. 18, 610-621 (2017).

84. Schiewer, M. J. \& Knudsen, K. E. Transcriptional Roles of PARP1 in Cancer. Mol Cancer Res 12, 1069-1080 (2014).

85. US Food and Drug Administration. FDA approves niraparib for first-line maintenance of advanced ovarian cancer. (2020). Available at: https://www.fda.gov/drugs/drugapprovals-and-databases/fda-approves-niraparib-first-line-maintenance-advancedovarian-cancer. (Accessed: 26th May 2021)

86. US Food and Drug Administration. FDA approves olaparib for germline BRCA-mutated metastatic breast cancer. (2018). Available at: https://www.fda.gov/drugs/resourcesinformation-approved-drugs/fda-approves-olaparib-germline-brca-mutated-metastaticbreast-cancer. (Accessed: 26th May 2021)

87. US Food and Drug Administration. FDA approves rucaparib for maintenance treatment of recurrent ovarian, fallopian tube, or primary peritoneal cancer. (2018). Available at: https://www.fda.gov/drugs/resources-information-approved-drugs/fda-approvesrucaparib-maintenance-treatment-recurrent-ovarian-fallopian-tube-or-primary-peritoneal. (Accessed: 26th May 2021)

88. US Food and Drug Administration. FDA approves talazoparib for gBRCAm HER2negative locally advanced or metastatic breast cancer. (2018). Available at: https://www.fda.gov/drugs/drug-approvals-and-databases/fda-approves-talazoparibgbrcam-her2-negative-locally-advanced-or-metastatic-breast-cancer. (Accessed: 26th May 2021)

89. Souzaki, M., Kubo, M., Kai, M., Kameda, C., Tanaka, H., Taguchi, T., Tanaka, M., Onishi, H. \& Katano, M. Hedgehog signaling pathway mediates the progression of non-invasive breast cancer to invasive breast cancer. Cancer Sci. 102, 373-381 (2011).

90. Liao, X., Siu, M. K. Y., Au, C. W. H., Wong, E. S. Y., Chan, Y., Ip, P. P. C., Ngan, Y. S. \& Cheung, A. N. Y. Aberrant activation of hedgehog signaling pathway in ovarian cancers: effect on prognosis, cell invasion and differentiation. Carcinogenesis 30, 131-140 (2009).

91. Gorodnova, T. V., Sokolenko, A. P., Ivantsov, A. O., Iyevleva, A. G., Suspitsin, E. N., Aleksakhina, S. N., Yanus, G. A., Togo, A. V., Maximov, S. Y. \& Imyanitov, E. N. High response rates to neoadjuvant platinum-based therapy in ovarian cancer patients carrying germ-line BRCA mutation. Cancer Lett. 369, 363-367 (2015).

92. Sakai, W., Swisher, E. M., Karlan, B. Y., Agarwal, M. K., Higgins, J., Friedman, C., Villegas, E., Jacquemont, C., Farrugia, D. J., Couch, F. J., Urban, N. \& Taniguchi, T. Secondary mutations as a mechanism of cisplatin resistance in BRCA2-mutated cancers. Nature 451, 1116-1120 (2008).

93. Sakai, W., Swisher, E. M., Jacquemont, C., Chandramohan, K. V., Couch, F. J., Langdon, S. P., Wurz, K., Higgins, J., Villegas, E. \& Taniguchi, T. Functional restoration of BRCA2 protein by secondary BRCA2 mutations in BRCA2-mutated ovarian carcinoma. Cancer Res. 69, 6381-6386 (2009).

94. Park, P. H., Yamamoto, T. M., Li, H., Alcivar, A. L., Xia, B., Wang, Y., Bernhardy, A. J., Turner, K. M., Kossenkov, A. V, Watson, Z. L., Behbakht, K., Casadei, S., Swisher, E. M., Mischel, P. S., Johnson, N. \& Bitler, B. G. Amplification of the Mutation-Carrying BRCA2 Allele Promotes RAD51 Loading and PARP Inhibitor Resistance in the Absence of Reversion Mutations. Mol. Cancer Ther. 19, 602-613 (2020).

95. Wang, C., Horiuchi, A., Imai, T., Ohira, S., Itoh, K., Nikaido, T., Katsuyama, Y. \& Konishi, 
I. Expression of BRCA1 protein in benign, borderline, and malignant epithelial ovarian neoplasms and its relationship to methylation and allelic loss of the BRCA1 gene. J. Pathol. 202, 215-223 (2004).

96. Shilpa, V., Bhagat, R., Premalata, C. S., Pallavi, V. R., Ramesh, G. \& Krishnamoorthy, L. BRCA1 promoter hypermethylation and protein expression in ovarian carcinoma - An Indian study. Tumor Biol. 35, 4277-4284 (2014).

97. Garg, K., Levine, D. A., Olvera, N., Dao, F., Bisogna, M., Secord, A. A., Berchuck, A., Cerami, E., Schultz, N. \& Soslow, R. A. BRCA1 immunohistochemistry in a molecularly characterized cohort of ovarian high-grade serous carcinomas. Am. J. Surg. Pathol. 37, 138-146 (2013).

98. Labidi-Galy, S. I., Papp, E., Hallberg, D., Niknafs, N., Adleff, V., Noe, M., Bhattacharya, R., Novak, M., Jones, S., Phallen, J., Hruban, C. A., Hirsch, M. S., Lin, D. I., Schwartz, L., Maire, C. L., Tille, J. C., Bowden, M., Ayhan, A., Wood, L. D., et al. High grade serous ovarian carcinomas originate in the fallopian tube. Nat. Commun. 8, 1-10 (2017).

99. Li, H. \& Durbin, R. Fast and accurate long-read alignment with Burrows-Wheeler transform. Bioinformatics 26, 589-595 (2010).

100. Van der Auwera, G. A., Carneiro, M. O., Hartl, C., Poplin, R., del Angel, G., LevyMoonshine, A., Jordan, T., Shakir, K., Roazen, D., Thibault, J., Banks, E., Garimella, K. V., Altshuler, D., Gabriel, S. \& DePristo, M. A. From fastQ data to high-confidence variant calls: The genome analysis toolkit best practices pipeline. Curr. Protoc. Bioinforma. 43, 11.10.1-11.10.33 (2013).

101. Dobin, A., Davis, C. A., Schlesinger, F., Drenkow, J., Zaleski, C., Jha, S., Batut, P., Chaisson, M. \& Gingeras, T. R. STAR: ultrafast universal RNA-seq aligner. Bioinformatics 29, 15-21 (2013).

102. Pertea, M., Kim, D., Pertea, G. M., Leek, J. T. \& Salzberg, S. L. Transcript-level expression analysis of RNA-seq experiments with HISAT, StringTie and Ballgown. Nat. Protoc. 11, 1650-1667 (2016).

103. Soneson, C., Love, M. I. \& Robinson, M. D. Differential analyses for RNA-seq: Transcriptlevel estimates improve gene-level inferences. F1000Research 4, 1521 (2016).

104. Robinson, M. D., Mccarthy, D. J. \& Smyth, G. K. edgeR: a Bioconductor package for differential expression analysis of digital gene expression data. Bioinformatics 26, 139140 (2010).

105. Robinson, M. D. \& Oshlack, A. A scaling normalization method for differential expression analysis of RNA-seq data. Genome Biol. 11, 3 (2010).

106. Lai, Z., Markovets, A., Ahdesmaki, M., Chapman, B., Hofmann, O., Mcewen, R., Johnson, J., Dougherty, B., Barrett, J. C. \& Dry, J. R. VarDict: A novel and versatile variant caller for next-generation sequencing in cancer research. Nucleic Acids Res. 44, 1-11 (2016).

107. Kim, S., Scheffler, K., Halpern, A. L., Bekritsky, M. A., Noh, E., Källberg, M., Chen, X., Kim, Y., Beyter, D., Krusche, P. \& Saunders, C. T. Strelka2: fast and accurate calling of germline and somatic variants. Nat. Methods 15, 591-594 (2018).

108. Cibulskis, K., Lawrence, M. S., Carter, S. L., Sivachenko, A., Jaffe, D., Sougnez, C., Gabriel, S., Meyerson, M., Lander, E. S. \& Getz, G. Sensitive detection of somatic point mutations in impure and heterogeneous cancer samples. Nat. Biotechnol. 31, 213-219 (2013).

109. Koboldt, D. C., Zhang, Q., Larson, D. E., Shen, D., McLellan, M. D., Lin, L., Miller, C. A., Mardis, E. R., Ding, L. \& Wilson, R. K. VarScan 2: Somatic mutation and copy number alteration discovery in cancer by exome sequencing. Genome Res. 22, 568-576 (2012).

110. Wang, K., Li, M. \& Hakonarson, H. ANNOVAR: Functional annotation of genetic variants from high-throughput sequencing data. Nucleic Acids Res. 38, 1-7 (2010).

111. Rosenthal, R., McGranahan, N., Herrero, J., Taylor, B. S. \& Swanton, C. deconstructSigs: 
Delineating mutational processes in single tumors distinguishes DNA repair deficiencies and patterns of carcinoma evolution. Genome Biol. 17, 31 (2016).

112. Wu, H.-X., Wang, Z.-X., Zhao, Q., Chen, D.-L., He, M.-M., Yang, L.-P., Wang, Y.-N., Jin, Y., Ren, C., Luo, H.-Y., Wang, Z.-Q. \& Wang, F. Tumor mutational and indel burden: a systematic pan-cancer evaluation as prognostic biomarkers. Ann. Transl. Med. 7, 640640 (2019).

113. Ioannidis, N. M., Rothstein, J. H., Pejaver, V., Middha, S., McDonnell, S. K., Baheti, S., Musolf, A., Li, Q., Holzinger, E., Karyadi, D., Cannon-Albright, L. A., Teerlink, C. C., Stanford, J. L., Isaacs, W. B., Xu, J., Cooney, K. A., Lange, E. M., Schleutker, J., Carpten, J. D., et al. REVEL: An Ensemble Method for Predicting the Pathogenicity of Rare Missense Variants. Am. J. Hum. Genet. 99, 877-885 (2016).

114. Quinlan, A. R. \& Hall, I. M. BEDTools: a flexible suite of utilities for comparing genomic features. Bioinformatics 26, 841-842 (2010).

115. Ronique Geoffroy, V., Herenger, Y., Kress, A., Stoetzel, C., Lie Piton, A., Lè Ne Dollfus, H. \& Muller, J. AnnotSV: an integrated tool for structural variations annotation. Bioinformatics 34, 3572-3574 (2018).

116. Kwon, A. T., Arenillas, D. J., Hunt, R. W. \& Wasserman, W. W. oPOSSUM-3: Advanced Analysis of Regulatory Motif Over-Representation Across Genes or ChIP-Seq Datasets. G3 2, 987-1002 (2012).

117. Haas, B. J., Dobin, A., Li, B., Stransky, N., Pochet, N. \& Regev, A. Accuracy assessment of fusion transcript detection via read-mapping and de novo fusion transcript assemblybased methods. Genome Biol. 20, 213 (2019). 


\section{Acknowledgments}

1278 The authors thank the patients and their families for participating in this study. We would also

1279 like to thank Lauren Schmucker, Caitlin Feltcher, Amy Ziober, Danielle Sharpe, Li-Ping Wang,

1280 Dr. Sean Carlin, and Heather Symecko for help with acquisition and staining of pathological

1281 specimens. We would like to thank Liza M. Dorfman for her assistance with whole exome

1282 sequencing and for her well-written protocols. This work was supported by the Tumor Tissue

1283 and Biospecimen Bank and the Next Generation Sequencing Core at the University of

1284 Pennsylvania (and in particular, we appreciated the help of Dr. Jonathan Schug). We would also

1285 like to thank Akoya Biosciences for their technical expertise regarding CODEX analyses. Lastly,

1286 the RNA sequencing pipeline used for this study was based on content from Dr. Dan Beiting's

1287 DIY Transcriptomics class at the University of Pennsylvania (Spring 2021), for which we thank

1288 him as well. This work was supported by the Basser Center for BRCA at the University of

1289 Pennsylvania, the Gray Foundation, the V Foundation for Cancer Research, and the Breast

1290 Cancer Research Foundation.

1291

1292

1293

1294

1295

1296

1297

1298

1299

1300

1301

1302

1303

\section{Author contributions}

J.B.S., K.N.M., and K.L.N conceived the study. J.B.S. performed the majority of DNA/RNA sequencing, bioinformatic analyses, and figure preparation (with exceptions below). J.B.S. and

K.L.N. primarily wrote the manuscript. D.P. performed all CODEX staining and imaging, and also prepared associated figures. B.W. performed sequence alignment, variant calling, and Sequenza analysis. J.P. assisted with calculation of HRD and aneuploidy scores and advised on numerous statistical analyses. K.D’A. performed DNA extraction and whole exome sequencing of germline specimens. A.H. and C.R assisted with clinical data curation. A.A.K. contributed to sample collection and protocols for RNA sequencing. K.N.M. optimized sequencing protocols and initiated tumor collection. J.S.S. performed DNA and RNA extractions and library preparations. W.Z., A.L.G., V.S., J.B., K.N.M., S.M.D., and K.L.N. all assisted in 
1304 identifying patients and specimens for the cohort. M.F. supervised collection and sectioning of

1305 FFPE specimens. A.N. performed pathological review of FFPE specimens, including IHC

1306 grading. S.M.D. offered clinical expertise. All authors contributing to editing the manuscript.

1307

\section{$1308 \quad$ Competing interests}

1309 S.M.D. has received honoraria from AstraZeneca.

1310

1311

1312

1313

1314

1315

1316

1317

1318

1319

1320

1321

1322

1323

1324

1325

1326

1327

1328

1329 Main Text Figures and Figure legends 
b

\begin{tabular}{|l|l}
\hline Breast \\
Ovary \\
BRCA1 \\
BRCA2 \\
\hline Primary \\
\hline Recurrence \\
Intact \\
$\begin{array}{l}\text { Heterozygous Loss } \\
\text { Homozygous } \\
\text { Deletion }\end{array}$ \\
\hline
\end{tabular}

$\square$ LOH

LOH Undetermined $\square$ nonLOH

Mutation Type Heterozygous LoF Heterozygous GoF No mutation \begin{tabular}{|l|l|}
\hline 14 &
\end{tabular} \begin{tabular}{|l|l|l|}
\hline 2 & \multicolumn{1}{|l|}{} & \\
\hline
\end{tabular} \begin{tabular}{|l|ll|l|}
\hline 3 & \multicolumn{1}{|l|}{} & & \\
\hline
\end{tabular} \begin{tabular}{|l|l|l|}
\hline 4 & \multicolumn{1}{|l|}{} & \\
\hline
\end{tabular} \begin{tabular}{|l|l|l|}
\hline 5 & \multicolumn{1}{|l|}{} & \\
\hline
\end{tabular} \begin{tabular}{l|l|l}
\hline 6 & 4
\end{tabular} \begin{tabular}{|l|l|l|}
\hline 7 & \multicolumn{1}{|l|}{} & \\
\hline
\end{tabular} \begin{tabular}{|l|lll|}
\hline 8 & \multicolumn{1}{|l|}{} & & \\
\hline
\end{tabular} \begin{tabular}{|l|l|l|l|}
\hline 9 & 2 & & \\
\hline
\end{tabular} \begin{tabular}{|l|l|l|l|}
\hline 10 & 4 \\
\hline
\end{tabular} \begin{tabular}{|l|l|l|l|}
\hline 11 & & & \\
\hline
\end{tabular} \begin{tabular}{|l|l|l|l|}
\hline 12 & \multicolumn{1}{|l|}{} & \\
\hline
\end{tabular} \begin{tabular}{|l|l|l|}
\hline 13 & $/$ \\
\hline
\end{tabular}

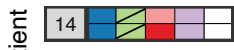

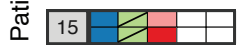
\begin{tabular}{|l|l|l|l|}
\hline 16 & E & & \\
\hline
\end{tabular} \begin{tabular}{|l||l|l|}
\hline 17 & \multicolumn{1}{|l|}{} & \\
\hline
\end{tabular} \begin{tabular}{|l|l|l|}
\hline 18 & \multicolumn{1}{|l|}{$\mid$} & \\
\hline
\end{tabular} $19 \%$ ${ }_{20}^{20}$ 21 \begin{tabular}{|l|l|l|}
\hline 22 & \multicolumn{1}{|l|}{} \\
\hline
\end{tabular} \begin{tabular}{l|l|l|}
\hline 23 & \multicolumn{1}{|l|}{} \\
\hline
\end{tabular} \begin{tabular}{|l|l|l|l|}
\hline 24 & \multicolumn{1}{|l|}{$\mid$} & & \\
\hline
\end{tabular} \begin{tabular}{|l|l|l|l|}
\hline 25 & & & \\
\hline
\end{tabular} \begin{tabular}{|l|l|l|}
\hline 26 & \multicolumn{1}{|l|}{} & \\
\hline
\end{tabular} \begin{tabular}{|l|l|l|}
\hline 27 & \multicolumn{1}{|l|}{$\mid$} & \\
\hline
\end{tabular}

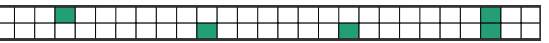

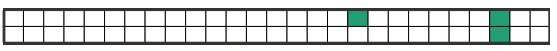

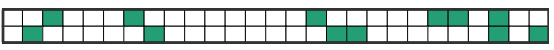

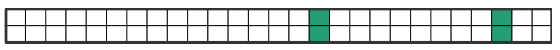

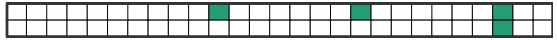

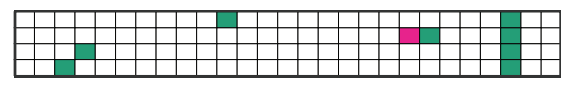

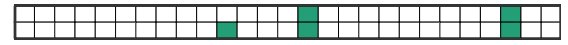

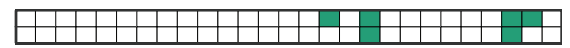
\begin{tabular}{|l|l|l|l|l|l|l|}
\hline \hline & & & & & &
\end{tabular}

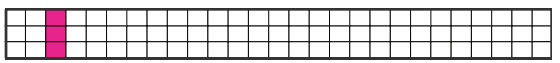

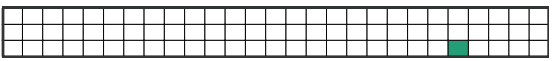

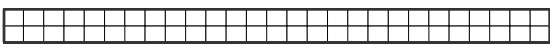

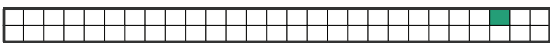

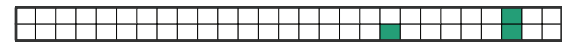

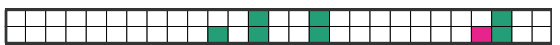

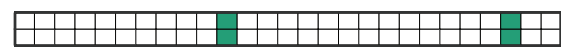

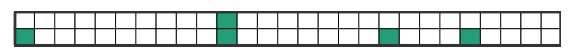

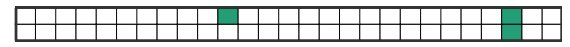

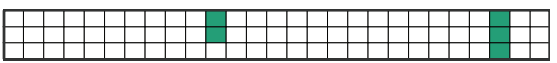
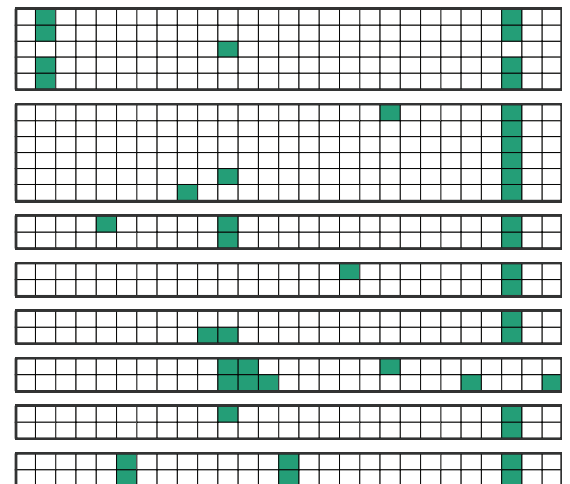

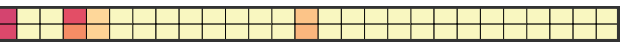

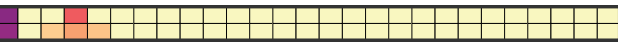

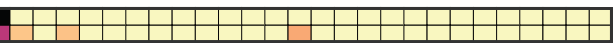

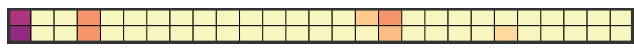

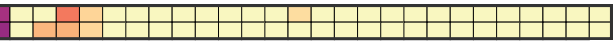

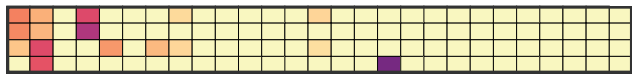

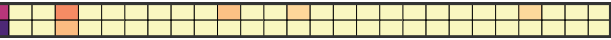

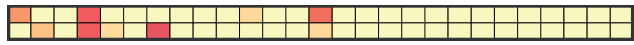

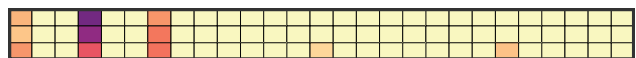

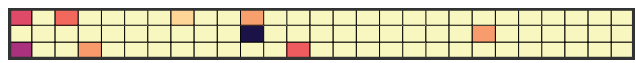

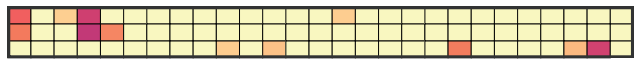

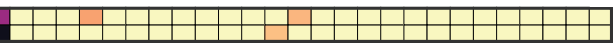

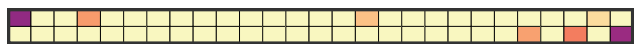

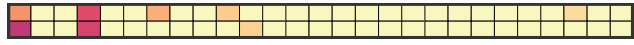

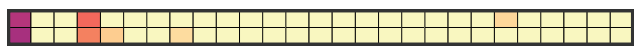

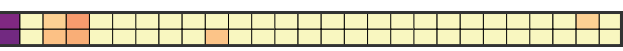

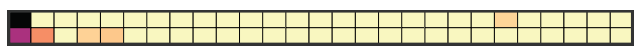

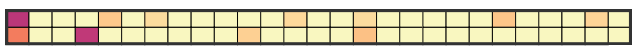

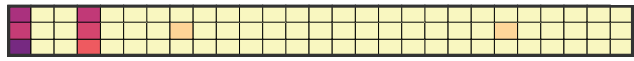
\begin{tabular}{|l|l|l|l|l|l|l|l|l|l|}
\hline \hline & & & & & & & & & \\
\hline
\end{tabular}

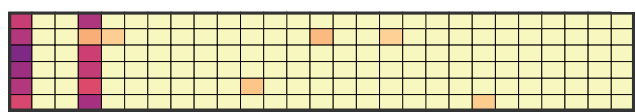

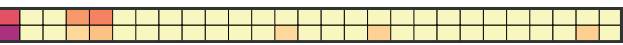

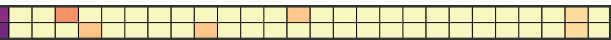

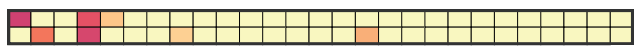

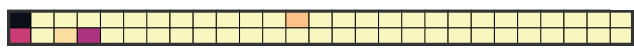

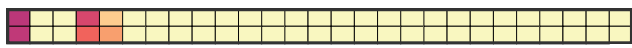

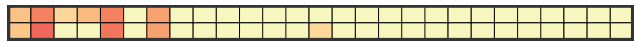

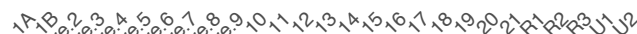

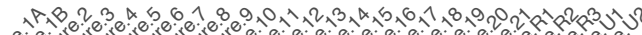

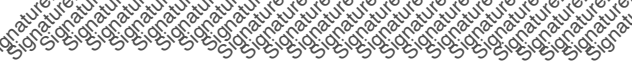

C
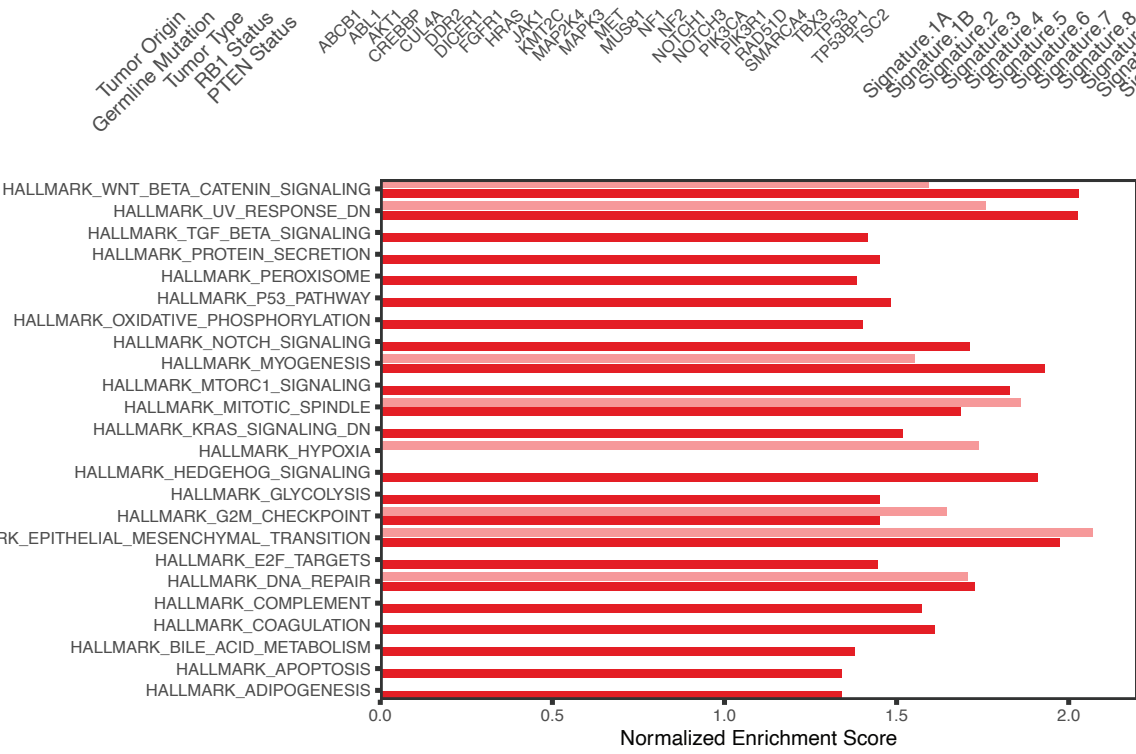

LoF Mutations in Primary Tumors LoF Mutations in Recurrences SENCHYMAL_TRANSITION HALLMARK_E2F_TARGETS HALLMARK DNA_REPAR HALLMARK_COAGULATION

HALLMARK_APOPTOSIS Normalized Enrichment Score

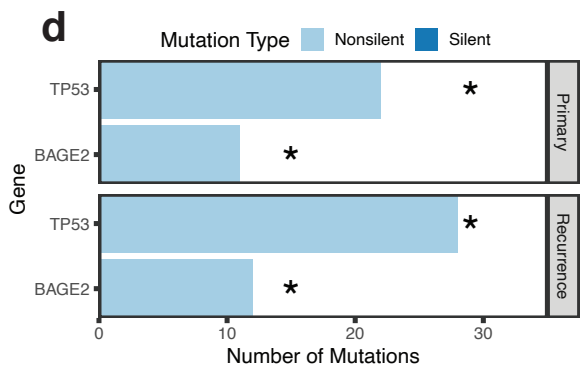

e

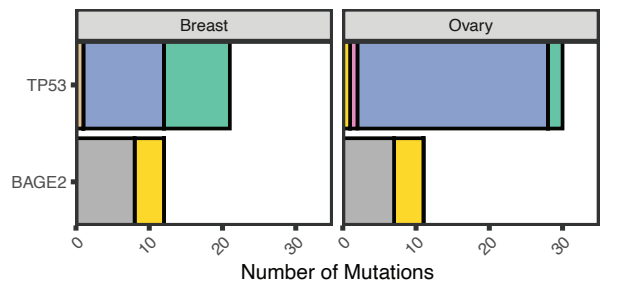

Variant Classification

of MutSigCV Mutations( ${ }^{*}$.maf)

$\square$ Frameshift

Nonsynonymous SNV (REVEL $>0.5$ )

Nonsynonymous SNV (REVEL $\leq 0.5)$

Splicing
Intronic 
Figure 1. Integrated analysis of somatic mutations by whole exome and targeted

1331 sequencing. A. Phenotype, BRCA1/2 allele-specific loss of heterozygosity (LOH), RB1 and

1332 PTEN status, and somatic mutations found in 67 paired primary and recurrent tumors (from 27

1333 patients) sequenced by whole exome sequencing (WES, $n=67$ ) and high-depth targeted

1334 sequencing $(n=44)$. Display is limited to genes with $\geq 1$ mutation with alternative allele fraction

$1335 \geq 0.05$ from targeted sequencing. Tumors are displayed in chronological order by patient, with

1336 the primary tumor at the top and latest recurrence at the bottom. B. Mutational signatures

1337 derived from WES mutations. C. Hallmark Gene Sets enriched in loss of function (LoF)

1338 mutations for primary and recurrent tumor groups (all FDR $q<0.25$ ). D. MutSigCV results ( ${ }^{\star} F D R$

$1339 \mathrm{q}<0.05)$. E. Variants contributing to MutSigCV results in $D$, by tumor type.

1340

1341

1342

1343

1344

1345

1346

1347

1348

1349

1350

1351

1352

1353

1354

1355 


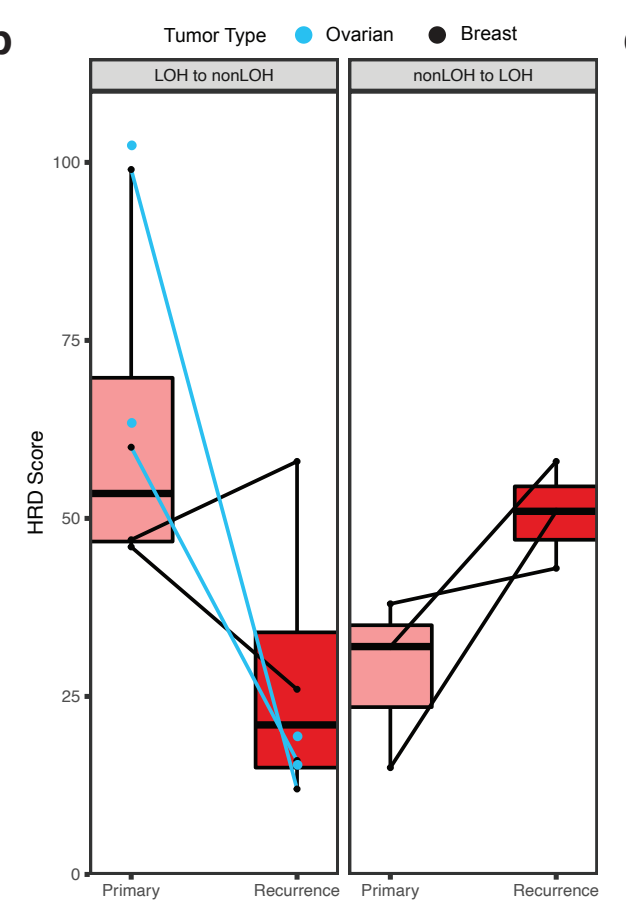

Lor $\mathrm{LOH}$
Undetermined $\square$ nonLOH

d

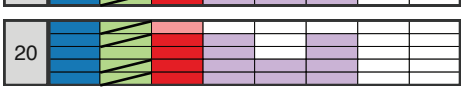

\begin{tabular}{|l|l|l|}
\hline 21 & \\
\hline
\end{tabular}

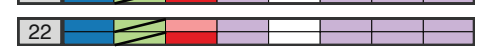

\begin{tabular}{|l|l|l|l|l|l|}
\hline 23 & & & & & \\
\hline
\end{tabular}

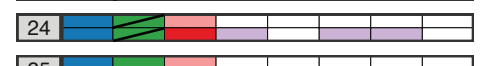

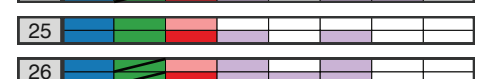
\begin{tabular}{|l|l|l|l|l|}
\hline 27 & \multicolumn{1}{|l|}{$\mid$} & & & \\
\hline
\end{tabular}

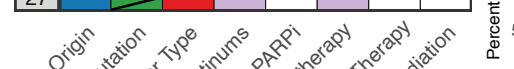
and e

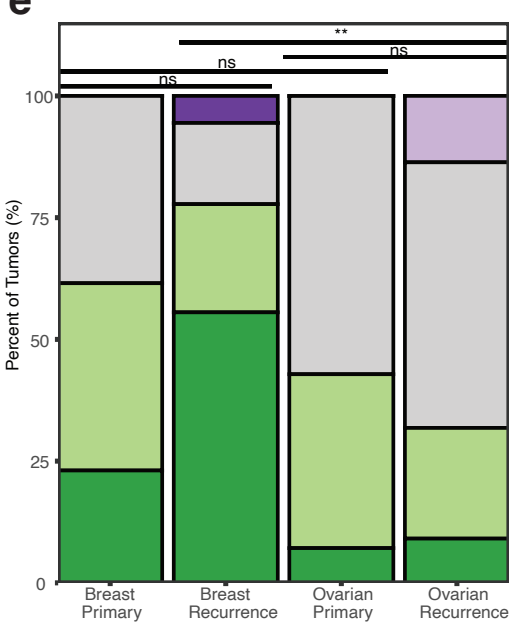

$\begin{array}{llll}\text { C } & 0.8 .4 & 0.62 & 0.8^{6.5 c o r e}\end{array}$
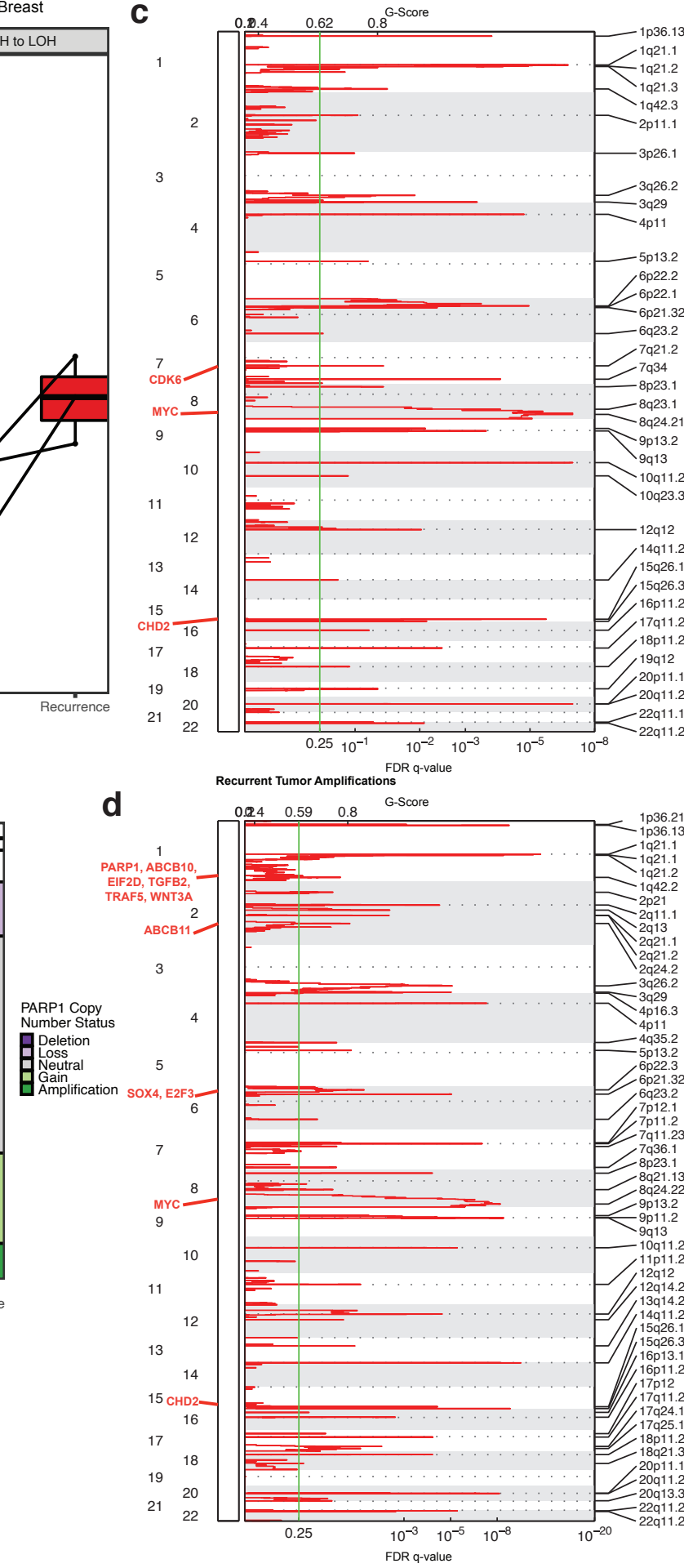

g

HALLMARK_UV_RESPONSE_UP -

HALLMARK_UV_RESPONSE_DN -

HALLMARK_TNFA_SIGNALING_VIA_NFKB -

HALLMARK_PEROXISOME

HALLMARK_PANCREAS_BETA_CELLS -

HALLMARK_NOTCH_SIGNALING

HALLMARK_MTORC1_SIGNALING

8 HALLMARK_INTERFERON_GAMMA_RESPONSE.

HALLMARK_INTERFERON_ALPHA_RESPONSE

HALLMARK_ANGIOGENESIS

HALLMARK_HEDGEHOG_SIGNALING -

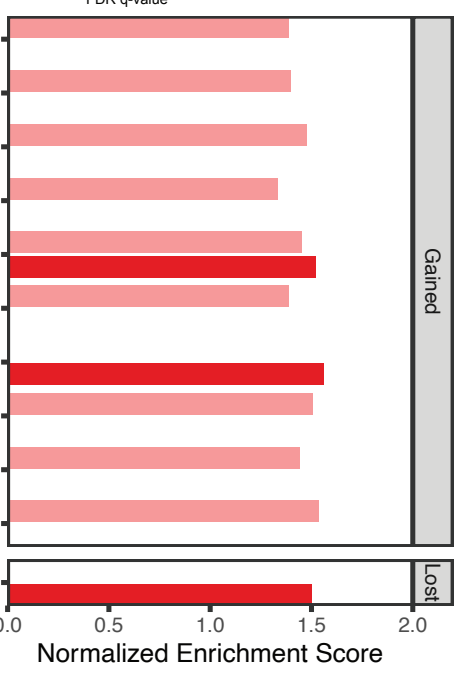

CNV in Primary Tumors CNV in Recurrences 
Figure 2. Genome-wide and gene-specific copy number variation. A. Allele specific loss of

1357 heterozygosity ( $\mathrm{LOH})$ of $B R C A 1 / 2$ in 67 paired primary and recurrent tumors, with treatment

1358 exposure. Tumors are displayed in chronological order by patient, with the primary tumor at the

1359 top and latest recurrence at the bottom. B. Comparison of HRD score for tumors with a change

1360 in LOH status from primary to recurrence. For one patient (Patient 6), who had multiple

1361 recurrences with $\mathrm{LOH}$, the first recurrence is displayed. C. GISTIC qplot for $90 \%$ confidence

1362 interval amplifications in primary tumors. D. GISTIC qplot for $90 \%$ confidence interval

1363 amplifications in recurrences. For $\mathrm{C}$ and $\mathrm{D}$, all highlighted genes have residual $\mathrm{q} \leq 0.05$. $\mathrm{E}$.

1364 PARP1 copy number by tumor in primary/recurrent cohort. Groupwise differences in average

1365 copy number were determined by Kruskal-Wallis test, followed by Dunn's test with Bonferroni

1366 correction $\left(\alpha=0.05,{ }^{* *} p<0.01\right)$. F. Segments of copy number gains and amplifications

1367 encompassing PARP1, by patient. For E and F, total copy number (Sequenza) was binned as

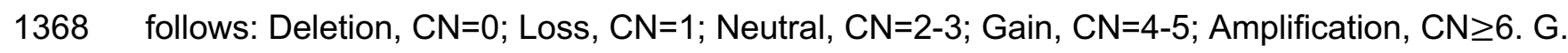

1369 Hallmark Gene Sets enriched in primary and recurrent tumor gains and amplifications $(\mathrm{CN} \geq 4$,

1370 top) vs. losses and deletions $(\mathrm{CN} \leq 1$, bottom) (all FDR $\mathrm{q}<0.25)$. 


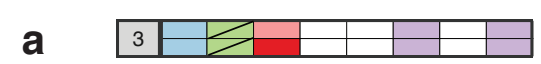

\begin{tabular}{|l|l|l|l|l|l|l|l|}
\hline 4 & \multirow{2}{*}{} & & & & & & \\
\hline
\end{tabular}

\begin{tabular}{|l|l|l|l|l|l|l|l|}
\hline 5 & & \multicolumn{1}{|c|}{} & & & & & \\
\hline
\end{tabular}

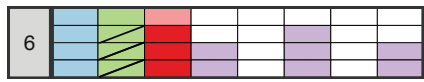

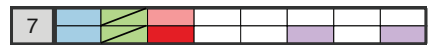

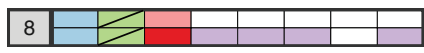

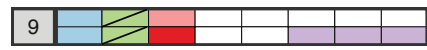

\begin{tabular}{|l|l|l|l|l|l|l|l|}
\hline 12 & & & & & & & \\
\hline
\end{tabular}

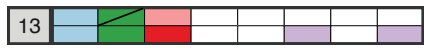

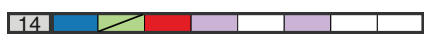

\begin{tabular}{|l|l|l|l|l|l|l|}
\hline 15 & $\mathrm{~S}^{2}$ & & & & & \\
\hline
\end{tabular}

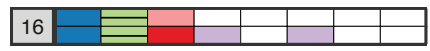

\begin{tabular}{|l|l|l|l|l|}
\hline 17 & \multicolumn{1}{|c|}{} & & & \\
\hline
\end{tabular}

\begin{tabular}{|l|l|l|l|l|l|}
\hline 18 & \multicolumn{1}{|l|}{} & & & & \\
\hline
\end{tabular}

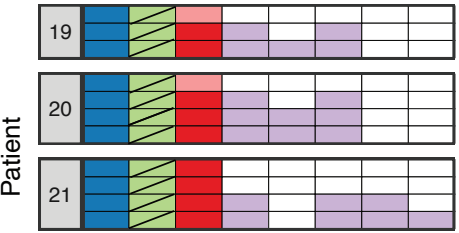

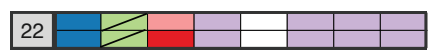

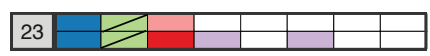
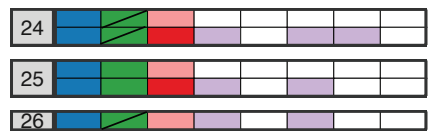

\begin{tabular}{|l|l|l|l|l|l|l|}
\hline 27 & $>$ & & & & & \\
\hline
\end{tabular}

\begin{tabular}{|l|l|l|l|l|l|l|}
\hline 28 & $<$ & & & & & \\
\hline
\end{tabular}

\begin{tabular}{|l|l|l|l|l|l|}
\hline 29 & $>$ & & & & \\
\hline
\end{tabular}

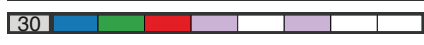

\begin{tabular}{|l|l|l|l|l|l|l|}
\hline 31 & $>$ & & & & & \\
\hline
\end{tabular}

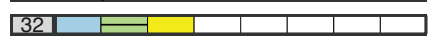

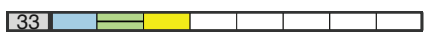

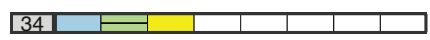

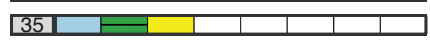

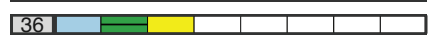

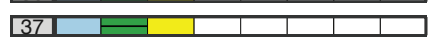

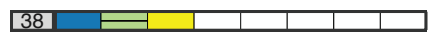

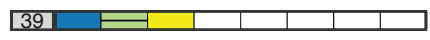

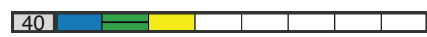

\begin{tabular}{|l|l|l|l|l|l|}
\hline 41 & L \\
\hline
\end{tabular}

\begin{tabular}{|l|l|l|l|l|l|}
\hline 42 & ह & \\
\hline
\end{tabular}

\begin{tabular}{|l|l|l|l|l|l|l|}
\hline 43 & L & \\
\hline 4
\end{tabular}
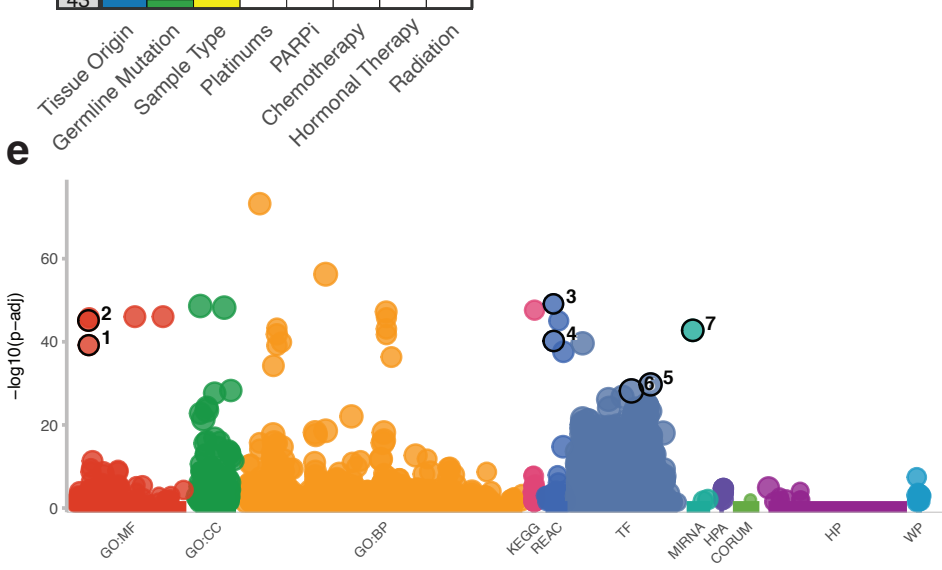

Terms Down in Tumor

\begin{tabular}{|l|l|l|l|l|l|}
\hline id & source & \multicolumn{1}{|c|}{ term_id } & \multicolumn{1}{|c|}{ term_name } & term_size & p_value \\
\hline 1 & GO:MF & GO:0004930 & G protein-coupled receptor activity & 840 & $6.3 e-40$ \\
\hline 2 & GO:MF & GO:0004888 & transmembrane signaling receptor activity & 1262 & $7.4 \mathrm{e}-46$ \\
\hline 3 & REAC & REAC:R-HSA-418555 & G alpha (s) signalling events & 535 & $7.5 \mathrm{e}-50$ \\
\hline 4 & REAC & REAC:R-HSA-388396 & GPCR downstream signalling & 1100 & $6.4 \mathrm{e}-41$ \\
\hline 5 & TF & TF:M07270_1 & Factor: TEF-3; motif: GNTATTTTT; match class: 1 & 4545 & $1.8 \mathrm{e}-30$ \\
\hline 6 & TF & TF:M02027_1 & Factor: PARP; motif: TTTCYN; match class: 1 & 12305 & $6.6 \mathrm{e}-29$ \\
\hline 7 & MIRNA & MIRNA:hsa-miR-335-5p & hsa-miR-335-5p & 2570 & $1.7 \mathrm{e}-43$ \\
\hline
\end{tabular}

C Ovarian Recurrences vs. Normal Fallopian Tube
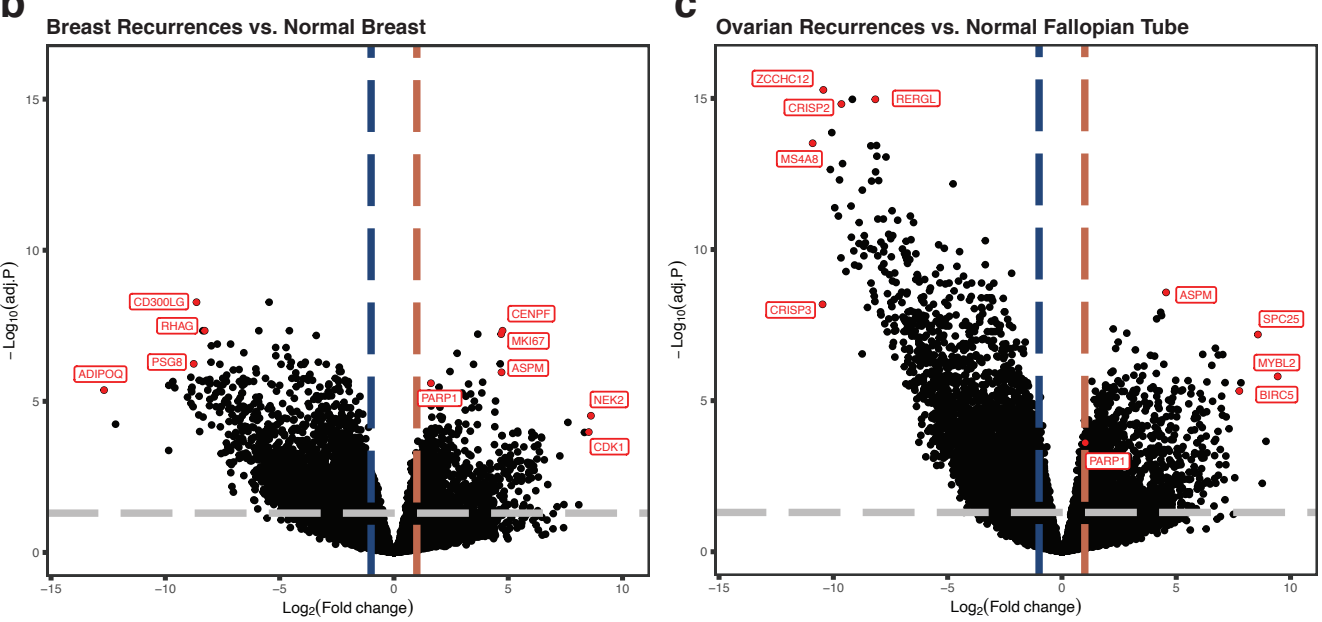

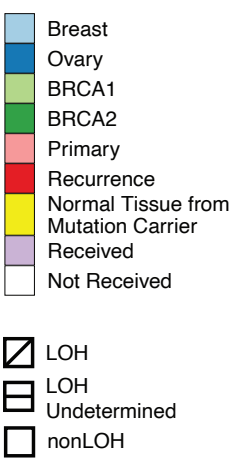

d HALLMARK_UNFOLDED_PROTEIN_RESPONSE -

HALLMARK_TNFA_SIGNALING_VIA_NFKB HALLMARK_SPERMATOGENESIS HALLMARK_REACTIVE_OXYGEN_SPECIES_PATHWAY HALLMARK_PI3K_AKT_MTOR_SIGNALING HALLMARK_P53_PATHWAY HALLMARK_OXIDATIVE_PHOSPHORYLATION HALLMARK_MYC_TARGETS_V2 HALLMARK_MYC_TARGETS_V1

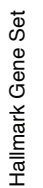
HALLMARK_MTORC1_SIGNALING HALLMARK_MITOTIC_SPINDLE HALLMARK_INTERFERON_GAMMA_RESPONSE HALLMARK_INTERFERON_ALPHA_RESPONSE ‘ HALLMARK_INFLAMMATORY_RESPONSE • HALLMARK_IL6_JAK_STAT3_SIGNALING

HALLMARK_IL2_STAT5_SIGNALING HALLMARK_GLYCOLYSIS HALLMARK_G2M_CHECKPOINT HALLMARK_ESTROGEN_RESPONSE_LATE HALLMARK_E2F_TARGETS HALLMARK_DNA_REPAIR HALLMARK_COMPLEMENT HALLMARK_APOPTOSIS HALLMARK_ALLOGRAFT_REJECTION

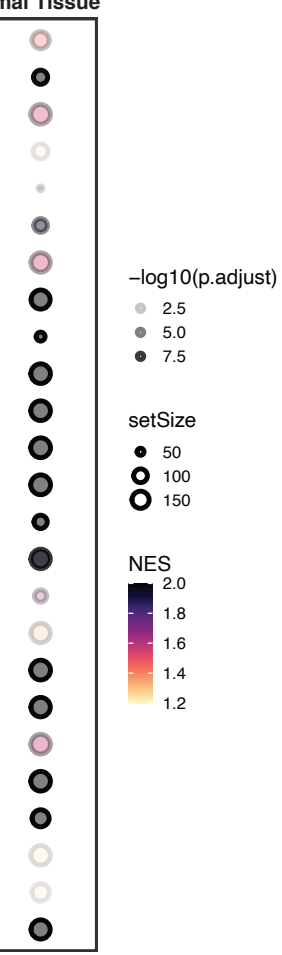

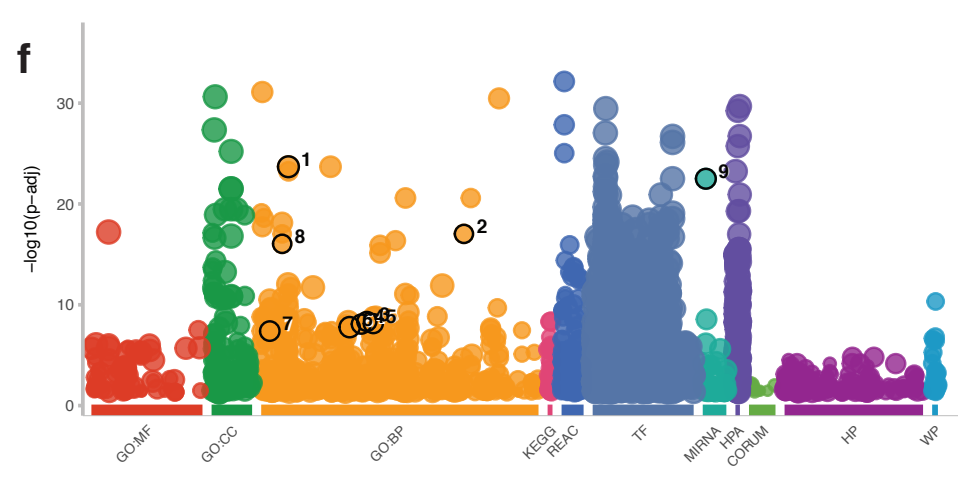

Terms Up in Tumor

\begin{tabular}{|l|l|l|l|l|l|}
\hline id & source & \multicolumn{1}{|c|}{ term_id } & \multicolumn{1}{c|}{ term_name } & term_size & P_value \\
\hline 1 & GO:BP & GO:0007049 & cell cycle & 1882 & $2.0 \mathrm{e}-24$ \\
\hline 2 & GO:BP & GO:0098813 & nuclear chromosome segregation & 268 & $9.3 \mathrm{e}-18$ \\
\hline 3 & GO:BP & GO:0042119 & neutrophil activation & 500 & $4.5 \mathrm{e}-09$ \\
\hline 4 & GO:BP & GO:0036230 & granulocyte activation & 506 & $8.2 \mathrm{e}-09$ \\
\hline 5 & GO:BP & GO:0043312 & neutrophil degranulation & 484 & $7.4 \mathrm{e}-09$ \\
\hline 6 & GO:BP & GO:0034097 & response to cytokine & 1238 & $1.7 \mathrm{e}-08$ \\
\hline 7 & GO:BP & GO:0002274 & myeloid leukocyte activation & 668 & $4.1 \mathrm{e}-08$ \\
\hline 8 & GO:BP & GO:0006260 & DNA replication & 283 & $9.0 \mathrm{e}-17$ \\
\hline 9 & MIRNA & MIRNA:hsa-miR-193b-3p & hsa-miR-193b-3p & 846 & $3.2 \mathrm{e}-23$ \\
\hline
\end{tabular}


1382 Figure 3. Global transcriptomic programs detected by RNA sequencing. A. 66 samples

1383 used for RNA sequencing. Tumors are displayed in chronological order by patient, with the 1384 primary tumor at the top and latest recurrence at the bottom. Patients 28-31 had recurrent 1385 tumors only. Patients 32-43 were BRCA1/2 mutation carriers with no prior history of cancer or 1386 cancer treatment; their samples are normal tissue from breast and fallopian tube. B. Differential 1387 gene expression in breast tumor recurrences vs. normal breast tissue. C. Differential gene 1388 expression in ovarian tumor recurrences vs. normal fallopian tube tissue. For B and C, a 1389 positive $\log _{2}$ (fold change) indicates genes with increased expression in recurrent tumors. 1390 Adjusted $p$ values were computed based on linear modeling of mean-variance trends (limma).

1391 D. Hallmark Gene Sets enriched in genes with increased expression in primary and recurrent 1392 breast and ovarian tumors compared to normal breast and fallopian tube tissue (all adj.p<0.05).

1393 E. Gene ontology terms enriched in genes with decreased expression in tumor vs. normal 1394 comparisons (module 1 [yellow] in Supplementary Fig. 8e). F. Gene ontology terms enriched 1395 genes with increased expression in tumor vs. normal comparisons (module 2 [blue] in 1396 Supplementary Fig. 8e). 
Figure 4. Gene fusions and isoform switching detected by RNA sequencing. A. Clinical

1408 characteristics of patients in which IGH-@, IGL-@, and MALAT1 fusions were identified.

1409 Tumors are displayed in chronological order by patient, with the primary tumor at the top and 1410 latest recurrence at the bottom. "1 fusion" refers to a translocation with one other gene. $\mathrm{B}$.

1411 Example of MALAT1-IGH-@ gene fusion IGV tracks from patient 9. Junction reads (red, middle

1412 track) represent split RNA-seq reads used to map the fusion breakpoint. Spanning reads (black,

1413 bottom track) represent paired-end reads of fragments that span, but do not directly overlap, the

1414 fusion breakpoint. C. Expression of total BRCA2 (all isoforms) with $95 \% \mathrm{Cl}$ in recurrences vs.

1415 normal samples. D. BRCA2 isoform usage (BRCA2 isoform expression normalized to total

1416 BRCA2 expression) in recurrences vs. normal samples; q values computed using DEXSeq

1417 within isoformSwitchAnalyzer. E. BRCA2 isoforms involved in isoform switching event. F.

1418 BRCA2 isoform expression by sample and group for entire RNA sequencing cohort. G. Survival

1419 curve for patients that expressed BRCA2-001/Short in any (primary or recurrent) breast tumor

1420 compared to those that did not. Survival proportions and $p$-value were calculated using a Cox

1421 proportional hazards model tested for significant associations with ER status, age at diagnosis,

1422 tumor stage at diagnosis, and patient recurrent status ( $\alpha=0.05$, see Methods). 
a

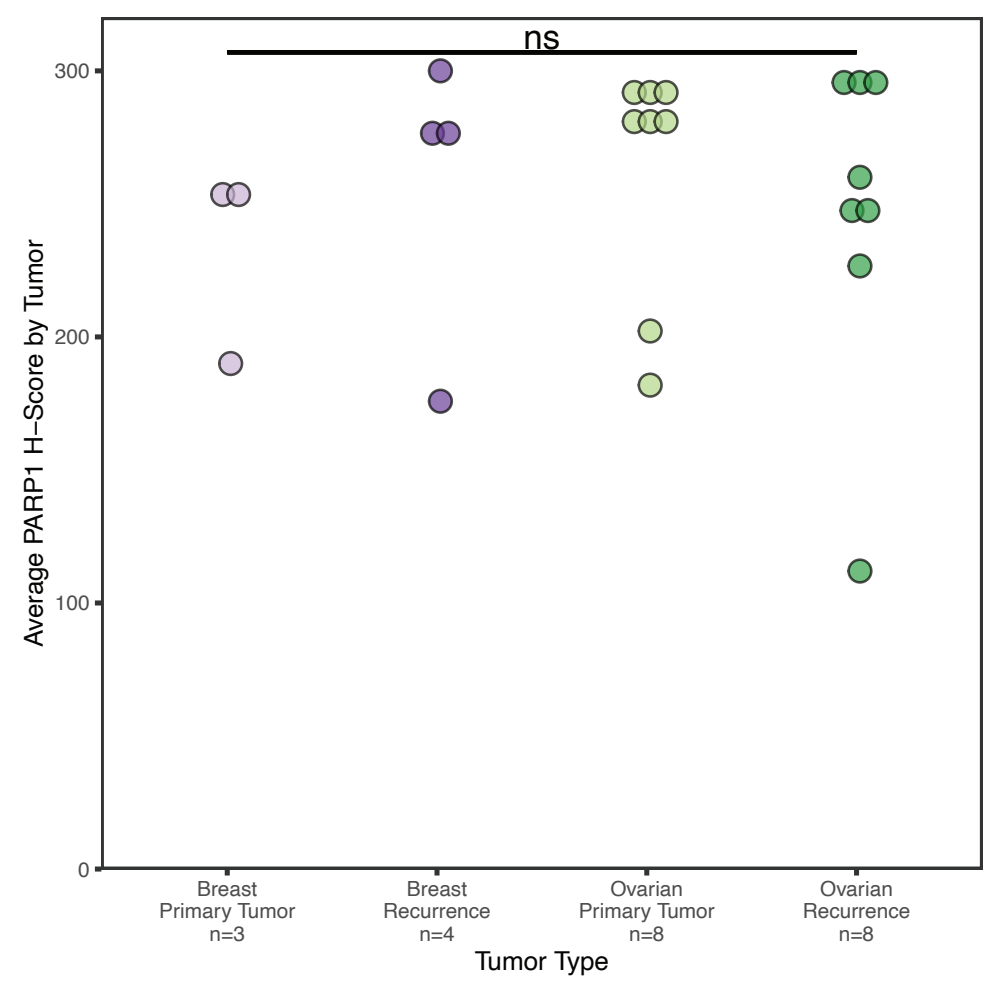

C

Breast Tumor

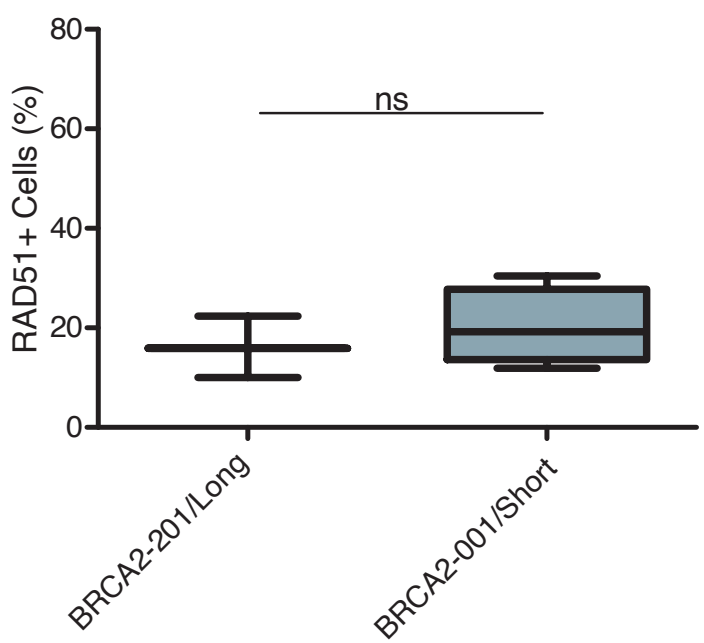

b

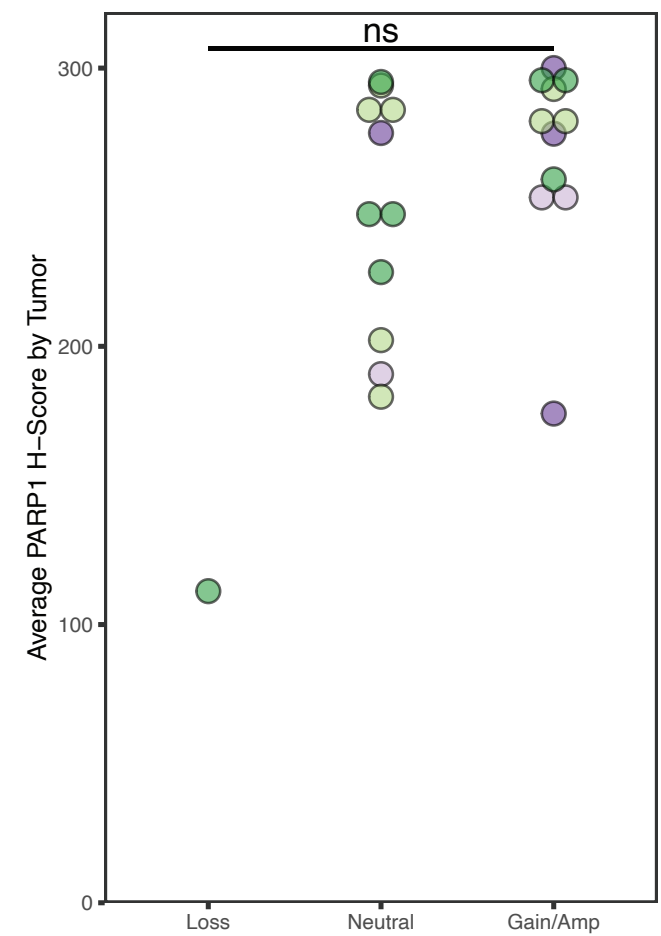

PARP1 Copy Number Status

d

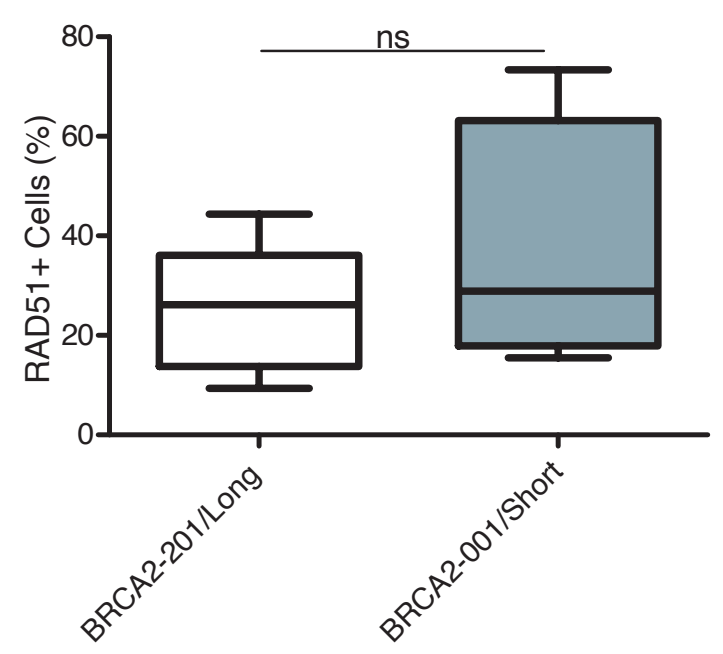

Tumor Type

- Primary Breast Tumor

- Recurrent Breast Tumor

- Primary Ovarian Tumor

- Recurrent Ovarian Tumor 
1432 Figure 5. Validation of prior results by PARP1 and RAD51 protein expression. A. PARP1

1433 nuclear positivity (average tumor $\mathrm{H}$-score) by tumor type, for 23 tumors in tissue microarrays. B.

1434 PARP1 nuclear positivity (average tumor $\mathrm{H}$-score) by PARP1 copy number status across all

1435 tumors. For A and B, groupwise differences were determined by Kruskal-Wallis test, followed by

1436 Dunn's test with Bonferroni correction $(\alpha=0.05)$. C. Percent of RAD51+ cells in primary $(n=3)$

1437 and recurrent $(n=4)$ breast tumors based on BRCA2 transcript usage. D. Percent of RAD51+

1438 cells in primary $(n=6)$ and recurrent $(n=7)$ ovarian tumors based on BRCA2 transcript usage. For

$1439 \mathrm{C}$ and D, groupwise differences were assessed by two-sided Wilcoxon rank sum test $(\alpha=0.05)$.

1440 Tumors without RNA-seq data $(n=2)$ or expressing both BRCA2 isoforms $(n=1)$ were excluded

1441 from this analysis.

1442

1443

1444

1445

1446

1447

1448

1449

1450

1451

1452

1453

1454

1455

1456 
Primary Ovarian Tumor

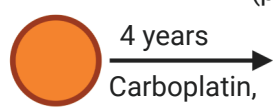

Recurrence 1

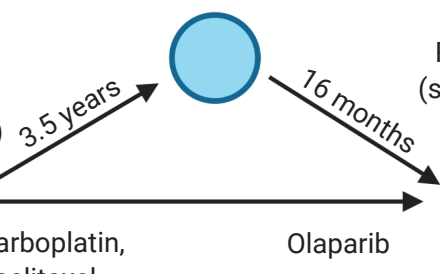

Recurrence 3

Recurrence 4

(supraclavicular (sternocleidomastoid (peritoneum)

muscle)
(sternocleidom

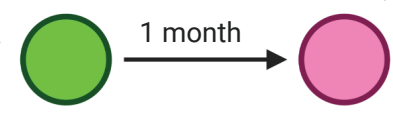

22 months $\underset{\text { Death }}{\longrightarrow}$ Paclitaxel

Paclitaxel,

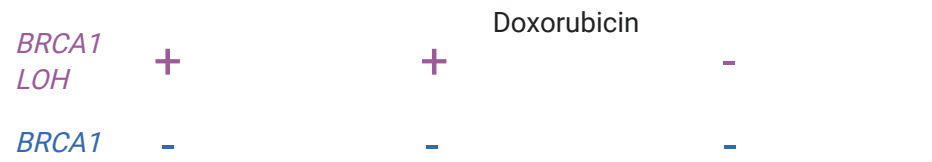

\section{$A B C B 10$,}

$A B C B 11+$

gains

BRCA2

isoform BRCA2-201/Long BRCA2-001/Short

b

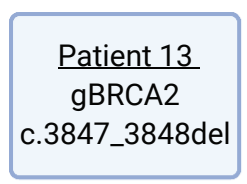

Primary Breast

Tumor

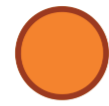

Recurrence

(supraclavicular lymph node)
Doxorubicin, Paclitaxel, Cyclophosphamide

Radiation

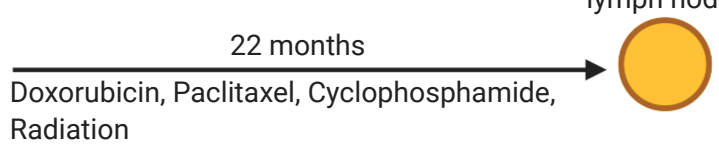

2 years

Carboplatin, Cisplatin, Trastuzumab, Pertuzumab, Palbociclib, Radiation, Non-hormonal chemo x3
Receptor status

$B R C A 2 \mathrm{LOH}$

$B R C A 2$ isoform

MYC status

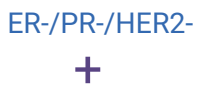

ER-/PR-/HER2-

$+$

BRCA2-201/Long

Neutral
ER-/PR-/HER2-

BRCA2-001/Short

Amplified

TMB, HRD score,

Aneuploidy score

Status of DNA

- Diploid MUTYH, RAD54L, RAD52

repair genes

- Copy number losses in MUTYH, RAD54L, RAD52

- RAD50 LOF mutation

C

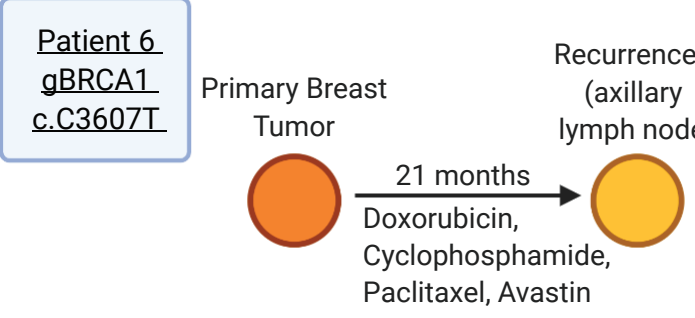

Receptor

status

BRCA1 LOH

$A B C B 10$,

$A B C B 11$ gains

BRCA2

isoform

ER-/PR-/HER2-

Status of DNA repair genes
- LOF mutations in ATR, ERCC2
ER-/PR-/HER2-

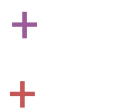

BRCA2-001/Short

- LOF mutations in ATR, ATM, XRCC1

e)

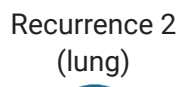

Recurrence 2 (lung)

Paclitaxel, Gemcitabine, Radiation

$+$

$+$ 
1457 Figure 6. Evolution of BRCA1/2 mutation-associated breast and ovarian tumors in three

1458 patients. A. Genetic and clinical features in Patient 20, a BRCA1 mutation carrier with ovarian

1459 cancer. B. Genetic and clinical features in Patient 13, a BRCA2 mutation carrier with breast

1460 cancer. C. Genetic and clinical features in Patient 6, a BRCA1 mutation carrier with breast

1461 cancer. For A-C, display is limited to tumors sequenced for this study. Copy number losses refer

1462 to genes with total copy number of 0 or 1 ; copy number gains refer to genes with total copy

1463 number $\geq 4$. Loss of function (LoF) mutations reported here are from high-depth targeted

1464 sequencing.

1465

1466

1467

1468

1469

1470

1471

1472

1473

1474

1475

1476

1477

1478

1479

1480

1481

1482 
Main Text Tables

Table 1: Characteristics of primary/recurrent patient cohort and tumors

\begin{tabular}{|c|c|c|c|}
\hline 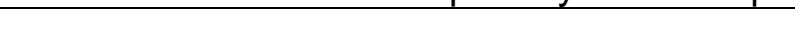 & Breast & Ovarian & Total \\
\hline Patients & 13 & 14 & 27 \\
\hline \multicolumn{4}{|l|}{ Tumors } \\
\hline \multirow{3}{*}{$\begin{array}{r}\text { Primary } \\
\text { Recurrent } \\
\text { Total }\end{array}$} & 13 & 14 & 27 \\
\hline & 18 & 22 & 40 \\
\hline & 31 & 36 & 67 \\
\hline \multicolumn{4}{|l|}{ Number of Recurrences Collected per Patient } \\
\hline 1 & 9 & 10 & 19 \\
\hline$>1$ & 4 & 4 & 8 \\
\hline \multicolumn{4}{|l|}{ Germline Mutation } \\
\hline BRCA1 & 9 & 10 & 19 \\
\hline BRCA2 & 4 & 4 & 8 \\
\hline \multicolumn{4}{|l|}{ ER Status of Primary Tumor } \\
\hline - & 4 & $\mathrm{~N} / \mathrm{A}$ & 4 \\
\hline ER- & 9 & $\mathrm{~N} / \mathrm{A}$ & 9 \\
\hline \multicolumn{4}{|l|}{ Stage at Diagnosis } \\
\hline I & 2 & 1 & 3 \\
\hline II & 8 & 3 & 11 \\
\hline III & 2 & 8 & 10 \\
\hline IV & 0 & 1 & 1 \\
\hline Unreported & 1 & 1 & 2 \\
\hline \multicolumn{4}{|l|}{ Treatment Received $^{a, b}$} \\
\hline Platinums & 3 & 14 & 17 \\
\hline $\mathrm{PARPi}^{\mathrm{C}}$ & 1 & 4 & 5 \\
\hline Chemotherapy & 13 & 14 & 27 \\
\hline Hormonal Therapy & 5 & 3 & 8 \\
\hline Radiation & 7 & 3 & 10 \\
\hline \multicolumn{4}{|c|}{$\begin{array}{l}{ }^{a} \text { Treatments are limited to reflect the number of patients from whom a post-treatment recurrence was } \\
\text { collected. } \\
\\
{ }^{b} \text { Treatment groups are not mutually exclusive; most patients received multiple treatments. } \\
{ }^{c} \text { PARPi = Poly (ADP-ribose) polymerase inhibitor }\end{array}$} \\
\hline
\end{tabular}




\section{Supplementary Files}

This is a list of supplementary files associated with this preprint. Click to download.

- SupplementaryData1.xlsx

- SupplementwithFigures07232021.pdf

- SupplementaryData6.xlsx

- SupplementaryData3.xIsx

- SupplementaryData9.xlsx

- SupplementaryData4.xIsx

- SupplementaryData11.xlsx

- SupplementaryData7.xIsx

- SupplementaryData5.xlsx

- SupplementaryData10.xlsx

- SupplementaryData2.xlsx

- SupplementaryData8.xlsx

- SupplementwithFigures07232021.pdf 